\title{
REDUCTION OF CONTROLLED LAGRANGIAN AND HAMILTONIAN SYSTEMS WITH SYMMETRY*
}

\author{
DONG EUI CHANG ${ }^{\dagger}$ AND JERROLD E. MARSDEN ${ }^{\ddagger}$
}

\begin{abstract}
We develop reduction theory for controlled Lagrangian and controlled Hamiltonian systems with symmetry. Reduction theory for these systems is needed in a variety of examples, such as a spacecraft with rotors, a heavy top with rotors, and underwater vehicle dynamics. One of our main results shows the equivalence of the method of reduced controlled Lagrangian systems and that of reduced controlled Hamiltonian systems in the case of simple mechanical systems with symmetry.
\end{abstract} alence

Key words. controlled Lagrangian systems, controlled Hamiltonian systems, reduction, equiv-

AMS subject classifications. 70Q05, 93C15, 34H05

DOI. $10.1137 / \mathrm{S} 0363012902412951$

1. Introduction. We develop the theory of symmetry reduction for the class of controlled Lagrangian (CL) and controlled Hamiltonian (CH) systems with symmetry and show the equivalence of the method of reduced CL systems and that of reduced $\mathrm{CH}$ systems for simple mechanical systems. The phrases "controlled Lagrangian" and "controlled Hamiltonian" systems were coined in [20], and their technical definition is recalled below. The concept of a $\mathrm{CH}$ system used here is the same as that of a "port-controlled Hamiltonian system" in [32]. We prefer to use the term "controlled Hamiltonian" in a way that is parallel to "controlled Lagrangian." The notion of an "implicit Hamiltonian system" is related to that of a CH system but is based on the more general notion of Dirac structures rather than symplectic and Poisson structures, and a type of reduction theory (different from what is given here) in that context was developed in [6]. The notion of an "implicit Lagrangian system" is developed, also using Dirac structures, in [35], and in future work we hope to explore the reduction theory of controlled implicit Hamiltonian and Lagrangian systems and the relation between them.

CL systems. We will begin by describing the Lagrangian side. To help design stabilizing controllers for mechanical systems whose equations are written in EulerLagrange form with control forces, the CL method was developed by a number of authors, including [3], [4], [7], [10], [11], [12], [13], [14], [15], [19], [20], [22], [23], [33]. In the study of CL systems, it is important to take into account external forces and control forces, including gyroscopic forces.

The main idea of the CL method is to proceed in the following steps:

1. Define a (feedback-transformation) equivalence relation among CL systems.

2. Given a CL system, find an equivalent CL system such that the energy of the second CL system has a minimum at the equilibrium of interest and the system is forced or controlled by a dissipative (plus gyroscopic) force.

* Received by the editors August 10, 2002; accepted for publication (in revised form) October 23, 2003; published electronically June 15, 2004. This research was partially supported by Caltech and by ONR contract N00014-02-1-0826.

http://www.siam.org/journals/sicon/43-1/41295.html

${ }^{\dagger}$ Centre Automatique et Systèmes, École Nationale Supérieure des Mines de Paris, 60, bd. SaintMichel, 75272 Paris CEDEX 06, France (dchang@cas.ensmp.fr).

${ }^{\ddagger}$ Control and Dynamical Systems 107-81, California Institute of Technology, Pasadena, CA 91125 (marsden@cds.caltech.edu). 
3. Using the energy as a Lyapunov function, obtain asymptotic stability of the equilibrium in the second CL system.

4. Since the two systems are equivalent, the equivalence relation gives an asymptotically stabilizing controller for the first system.

$\mathrm{CH}$ systems. A similar approach has been taken on the Hamiltonian side, too, where the main governing equations are in Hamiltonian form (see [5], [9], [32], [34] and references therein).

Equivalence. There have been some recent studies concerning the equivalence of both methods. First of all, [5] showed that the $\mathrm{CH}$ method is more general than the CL method for simple mechanical systems and used a limited definition of CL systems. Then [20] extended the theory of CL systems and showed the full equivalence of the method of CL systems with the method of $\mathrm{CH}$ systems for simple mechanical systems.

Symmetry. The main goal of the present paper is to include symmetry in the discussion. We do so in a systematic and general way. In previous works, symmetry was considered in certain cases (see, for instance, [15], [23], and [26]), but a comprehensive theory has been lacking.

In this paper, we define the notion of $G$-invariant CL and CH systems and develop a reduction theory for them. In addition, we trace through the equivalence of the CL and $\mathrm{CH}$ systems under the reduction process.

Reduction theory. Reduction theory for mechanical systems has been studied both on the Lagrangian side and on the Hamiltonian side. It has proven to be a powerful tool for studying the dynamics, stability, and control of many mechanical systems, such as rigid body and spacecraft systems, underwater vehicles, heavy tops, and fluid systems.

On the Lagrangian side, one reduces variational principles, an idea due to [29] and [30]. A comprehensive account of this theory is given in [17]. On the other hand, on the Hamiltonian side, one reduces symplectic and Poisson structures. See, for instance, [1], [27], and [28] and references therein. We shall merge these ideas with those of CL and $\mathrm{CH}$ systems to develop the notions and basic properties of reduced $\mathrm{CL}$ and $\mathrm{CH}$ systems.

Equivalence and reduction. Once we have developed the reduction theory, we will be in a position to define an equivalence relation among $G$-invariant CL (resp., $\mathrm{CH})$ systems; in this context, we show that two reduced CL (resp., CH) systems are equivalent if and only if their unreduced $G$-invariant CL (resp., CH) systems are equivalent. This is given in Theorems 2.10 and 3.9.

We also show that this reduced equivalence relation is a feedback-transform equivalence relation (see Theorems 2.11 and 3.10). Based on these results and the equivalence of the methods of CL and CH systems for simple mechanical systems proven in [20], we prove that the method of reduced CL systems and that of reduced $\mathrm{CH}$ systems are equivalent for reduced simple mechanical systems (this is given in Theorem 4.3).

Example. In the final section, we review the example of a satellite controlled via a spinning rotor and show that the Euler-Poincaré matching conditions derived in [8] and [15] directly in the reduced context can be obtained in a natural way through the reduction process. We study the stabilization of the heavy top with rotors, using the reduced CL method as an illustration.

2. Reduction of CL systems with symmetry. We begin this section with some general notation, then we introduce the notion of a CL system with symmetry, and finally study the theory of reduction for such systems. 
We first summarize some general notation that will be used. We refer to [2], [17], [25], and [28] for more details and background information.

Manifolds and bundles. Let $Q$ be the configuration manifold, and let $\tau_{Q}: T Q \rightarrow Q$ and $\pi_{Q}: T^{*} Q \rightarrow Q$ be the tangent bundle projection and the cotangent bundle projection, respectively. The second order tangent bundle $\tau_{Q}^{(2)}: T^{(2)} Q \rightarrow Q$ is defined as follows. For $\bar{q} \in Q$, elements of $T_{q}^{(2)} Q$ are equivalence classes of curves in $Q$, the equivalence relation being defined as follows: two curves $q_{i}(t), i=1,2$, with $q_{1}(\bar{t})=q_{2}(\bar{t})=\bar{q}$, are equivalent, by definition, if and only if in any local chart we have $q_{1}^{(l)}(\bar{t})=q_{2}^{(l)}(\bar{t})$ for $l=1,2$, where $q^{(l)}(t)$ denotes the derivative of order $l$. The second order bundle has local coordinates given by $\left(q^{i}, \dot{q}^{i}, \ddot{q}^{i}\right)$ and may be thought of as a subbundle of the second tangent bundle $T T Q$ via the embedding $\left(q^{i}, \dot{q}^{i}, \ddot{q}^{i}\right) \mapsto\left(q^{i}, \dot{q}^{i}, \dot{q}^{i}, \ddot{q}^{i}\right)$. As is explained in [28], the second order tangent bundle is the basic space on which the Euler-Lagrange operator of mechanics is defined; in fact, given a Lagrangian $L$, the associated Euler-Lagrange operator $\mathcal{E} \mathcal{L}$ induces a bundle $\operatorname{map} \mathcal{E} \mathcal{L}(L): T^{(2)} Q \rightarrow T^{*} Q$.

For a manifold $M, \mathcal{F}(M)$ denotes the set of smooth real-valued functions on $M$.

Symmetry groups. Let $G$ be a Lie group acting (on the left) on $Q$ freely and properly so that $\pi_{G}(Q): Q \rightarrow Q / G$ becomes a principal bundle. The tangent (resp., cotangent) lift of the action of $G$ on $Q$ defines an action of $G$ on $T Q$ (resp., $T^{*} Q$ ), which is automatically free and proper as well, so that the maps $\tau_{/ G}: T Q \rightarrow T Q / G$ (resp., $\pi_{/ G}: T^{*} Q \rightarrow T^{*} Q / G$ ) also define principal bundles. When $M$ is a manifold on which $G$ acts, we let $[m]_{G}$ denote an equivalence class of $m \in M$ in the quotient space $M / G$. Even though we do not explicitly denote the manifold $M$ in this notation, it will be clear which manifold is meant from the context. The space $T Q / G$ becomes a vector bundle with base $Q / G$ by inheriting the vector bundle structure of $T Q$ as follows:

$$
\left[u_{q}\right]_{G}+\lambda\left[v_{q}\right]_{G}=\left[u_{q}+\lambda v_{q}\right]_{G},
$$

where $\lambda \in \mathbb{R} ; u_{q}, v_{q} \in T_{q} Q$; and $\left[u_{q}\right]_{G},\left[v_{q}\right]_{G}$ are their equivalence classes in the quotient space $T Q / G$. The fiber $(T Q / G)_{x}$ is isomorphic, as a vector space, to $T_{q} Q$ for each $x=[q]_{G} \in Q / G, q \in Q$ (see Lemma 2.4 .1 in [17]). In the same manner, the space $T^{*} Q / G$ becomes a vector bundle with base $Q / G$.

Vertical lifts. Let $V$ be a vector bundle over a manifold $Q$. The vertical lift of a vector $w_{q} \in V_{q}$ along the vector $v_{q} \in V_{q}$ is the vector, $\operatorname{vlift}_{v_{q}}\left(w_{q}\right) \in T_{v_{q}} V$, defined by

$$
\operatorname{vlift}_{v_{q}}\left(w_{q}\right)=\left.\frac{d}{d t}\right|_{t=0}\left(v_{q}+t w_{q}\right) .
$$

The vertical lift of a fiber-preserving map $F: V \rightarrow V$ is a section, $\operatorname{vlift}(F): V \rightarrow$ $T V$, defined by

$$
\operatorname{vlift}(F)\left(v_{q}\right)=\operatorname{vlift}_{v_{q}}\left(F\left(v_{q}\right)\right) .
$$

The vertical lift of a subbundle $W$ of $V$ is the subbundle of $T V$ defined by

$$
\operatorname{vlift}(W)=\left\{\operatorname{vlift}_{v_{q}}\left(w_{q}\right) \mid v_{q} \in V_{q}, w_{q} \in W_{q}, q \in Q\right\} .
$$

$C L$ systems. We next recall the definition of a CL system from [20].

Definition 2.1. A controlled Lagrangian (CL) system is a triple $(L, F, W)$, where the function $L: T Q \rightarrow \mathbb{R}$ is the Lagrangian, the fiber-preserving map $F$ : 
$T Q \rightarrow T^{*} Q$ is the (external) force map, and the subbundle $W$ of $T^{*} Q$ is called the control subbundle. When one chooses a feedback control law $u: T Q \rightarrow W$, the triple $(L, F, u)$ will denote the closed-loop CL system.

Euler-Lagrange operator. The Euler-Lagrange operator $\mathcal{E} \mathcal{L}$ assigns to a Lagrangian $L: T Q \rightarrow \mathbb{R}$ a bundle map $\mathcal{E} \mathcal{L}(L): T^{(2)} Q \rightarrow T^{*} Q$, which may be written in local coordinates (and using the index summation convention) as

$$
\mathcal{E} \mathcal{L}(L)(q, \dot{q}, \ddot{q})=\left(\frac{d}{d t} \frac{\partial L}{\partial \dot{q}^{i}}(q, \dot{q})-\frac{\partial L}{\partial q^{i}}(q, \dot{q})\right) \mathbf{d} q^{i},
$$

in which it is understood that one regards the first term on the right-hand side as a function on the second order tangent bundle $T^{(2)} Q$ by formally applying the chain rule and replacing $d q / d t$ by $\dot{q}$ everywhere. The equations of motion of a CL system $(L, F, W)$ with a choice of feedback control $u: T Q \rightarrow W$ are written as

$$
\mathcal{E} \mathcal{L}(L)(q, \dot{q}, \ddot{q})=F(q, \dot{q})+u(q, \dot{q}),
$$

which may be derived from the Lagrange-d'Alembert principle.

$C L$ systems with symmetry. We next define the notion of a $G$-invariant CL system on $T Q$ and the associated notion of a reduced CL system on $T Q / G$, where $G$ is a Lie group acting on $Q$.

Definition 2.2. Let $G$ be a Lie group action on $Q$. A $G$-invariant controlled Lagrangian $(G$-CL) system is a $C L$ system, $(L, F, W)$, where $L$ is a $G$-invariant Lagrangian, $F$ is a $G$-equivariant force map, and $W$ is a $G$-invariant subbundle of $T^{*} Q$.

Reduction of CL systems with symmetry. Now we can define the notion of a reduced CL system.

Definition 2.3. A reduced controlled Lagrangian (RCL) system is a triple $(l, f, U)$, where the function $l: T Q / G \rightarrow \mathbb{R}$ is called the reduced Lagrangian, the fiber-preserving map $f: T Q / G \rightarrow T^{*} Q / G$ is called the reduced force map, and the subbundle $U$ of $T^{*} Q / G$ is called the reduced control subbundle. A feedback control for an $R C L$ system is a fiber-preserving map of $T Q / G$ into $U$.

Suppose that we are given a $G$-CL system $(L, F, W)$. The $G$-invariance of $L$ induces the reduced Lagrangian $l$ on $T Q / G$ satisfying

$$
l \circ \tau_{/ G}=L .
$$

The equivariance of $F$ induces the reduced force map $[F]_{G}: T Q / G \rightarrow T^{*} Q / G$ satisfying

$$
[F]_{G} \circ \tau_{/ G}=\pi_{/ G} \circ F .
$$

Similarly, a $G$-invariant control subbundle $W$ induces a reduced control subbundle $W / G$ in a natural way; namely, we have $W=\pi_{/ G}^{-1}(W / G)$.

These considerations lead to the following definition.

Definition 2.4. The RCL system of a $G$-CL system $(L, F, W)$ is the triple $\left(l,[F]_{G}, W / G\right)$, where $l$ is the reduced Lagrangian satisfying $(2.4)$ and $[F]_{G}$ is the reduced force satisfying (2.5).

One may ask whether there exists a $G$-CL system on $T Q$ when one is given a RCL system on $T Q / G$. The following proposition proves its existence and uniqueness.

Proposition 2.5. Given a $R C L$ system $(l, f, U)$, there is a unique $G$-CL system $(L, F, W)$ whose $R C L$ system is $(l, f, U)$. 
Proof. Define $L$ by (2.4). Define a force map $F$ on $T Q$ as follows: for $v_{q}, w_{q} \in T_{q} Q$,

$$
\left\langle F\left(v_{q}\right), w_{q}\right\rangle=\left\langle f \circ \tau_{/ G}\left(v_{q}\right), \tau_{/ G}\left(w_{q}\right)\right\rangle .
$$

One can check the $G$-equivariance of $F$. One can also check that relation (2.6) defines the unique fiber-preserving map $F$ of $T Q$ to $T^{*} Q$. Let $W:=\pi_{/ G}^{-1}(U)$. By construction, $(L, F, W)$ is the unique $G$-CL system whose RCL system is $(l, f, U)$.

By Proposition 2.5, we can write an arbitrary RCL in the form of the RCL of a $G$-CL without loss of generality. Additionally, the proof of Proposition 2.5 establishes the following assertion: given a fiber-preserving map $f: T Q / G \rightarrow T^{*} Q / G$, there exists the unique fiber-preserving map $F: T Q \rightarrow T^{*} Q$ satisfying

$$
f \circ \tau_{/ G}=\pi_{/ G} \circ F \text {. }
$$

Reduced equations of motion. Given a $G$-CL system $(L, F, W)$, the $G$-invariance of $L$ implies the $G$-equivariance of the bundle map $\mathcal{E} \mathcal{L}(L): T^{(2)} Q \rightarrow T^{*} Q$, which induces a quotient map

$$
\mathcal{R E} \mathcal{L}(l):=[\mathcal{E} \mathcal{L}(L)]_{G}: T^{(2)} Q / G \rightarrow T^{*} Q / G,
$$

which depends only on the reduced Lagrangian $l$ on $T Q / G$ induced from $L$. The operator $\mathcal{R E} \mathcal{L}$ is called the reduced Euler-Lagrange operator. The equations of motion of an RCL $\left(l,[F]_{G}, W / G\right)$ with a choice of control $[u]_{G}: T Q / G \rightarrow W / G$ are given by

$$
\mathcal{R E} \mathcal{L}(l)\left([q, \dot{q}, \ddot{q}]_{G}\right)=[F]_{G}\left([q, \dot{q}]_{G}\right)+[u]_{G}\left([q, \dot{q}]_{G}\right) .
$$

To write computable equations of $\mathcal{R E} \mathcal{L}$, one normally chooses a principal connection on the principal bundle $Q \rightarrow Q / G$ to identify the quotient bundles,

$$
\begin{aligned}
& T Q / G \text { with } T(Q / G) \oplus \tilde{\mathfrak{g}}, \\
& T^{(2)} Q / G \text { with } T^{(2)}(Q / G) \times_{Q / G} 2 \tilde{\mathfrak{g}},
\end{aligned}
$$

and

$$
T^{*} Q / G \text { with } T^{*}(Q / G) \oplus \tilde{\mathfrak{g}}^{*},
$$

where $\tilde{\mathfrak{g}}:=Q \times_{G} \mathfrak{g}$ is the associated adjoint bundle, $\tilde{\mathfrak{g}}^{*}:=Q \times_{G} \mathfrak{g}^{*}$ is the associated coadjoint bundle, $T^{(2)}(Q / G) \times_{Q / G} 2 \tilde{\mathfrak{g}}$ is the product bundle over $Q / G, 2 \tilde{\mathfrak{g}}:=\tilde{\mathfrak{g}} \oplus \tilde{\mathfrak{g}}$, and $\oplus$ is the Whitney sum (see Lemmas 2.4.2 and 3.2.2 in [17]). For example, a principal connection $A: T Q \rightarrow \mathfrak{g}$ on the principal bundle $\pi: Q \rightarrow Q / G$ induces the bundle isomorphism $\alpha_{A}: T Q / G \rightarrow T(Q / G) \oplus \tilde{\mathfrak{g}}$ as follows:

$$
\alpha_{A}\left([q, \dot{q}]_{G}\right)=T \pi(q, \dot{q}) \oplus[q, A(q, \dot{q})]_{G} .
$$

With these identifications, $\mathcal{R E} \mathcal{L}$ induces the Lagrange-Poincaré operator $\mathcal{L P}$ as follows: for a reduced Lagrangian $l$,

$$
\mathcal{L P}(l): T^{(2)}(Q / G) \times_{Q / G} 2 \tilde{\mathfrak{g}} \rightarrow T^{*}(Q / G) \oplus \tilde{\mathfrak{g}}^{*} .
$$

Hence, the reduced Euler-Lagrange operator $\mathcal{R E} \mathcal{L}$ may be replaced by the LagrangePoincaré operator $\mathcal{L P}$ in this paper as far as one chooses a connection on $Q \rightarrow Q / G$. Further details may be found in [17]. 
Let us give the local coordinate expression of the Lagrange-Poincare operator $\mathcal{L P}$ induced from a connection $A$ on the principal bundle $Q \rightarrow Q / G$. This will be used in section 5. We choose a local trivialization of the bundle $Q \rightarrow Q / G$ to be $X \times G \rightarrow X$, where $X$ is an open subset of $\mathbb{R}^{r}$ with $r=\operatorname{dim}(Q / G)$. Then, at any tangent vector $(x, g, \dot{x}, \dot{g}) \in T_{(x, g)}(X \times G)$, we have

$$
A(x, g, \dot{x}, \dot{g})=\operatorname{Ad}_{g}\left(A_{e}(x) \cdot \dot{x}+\xi\right),
$$

where $A_{e}$ is the $\mathfrak{g}$-valued 1 -form on $X$ defined by $A_{e}(x) \cdot \dot{x}=A(x, e, \dot{x}, 0)$ and $\xi=g^{-1} \dot{g}$. The bundle isomorphism $\alpha_{A}$ in this case becomes

$$
\alpha_{A}\left([x, g, \dot{x}, \dot{g}]_{G}\right)=(x, \dot{x}) \oplus(x, \Omega),
$$

where $\Omega=A_{e}(x) \cdot \dot{x}+\xi$ and we choose a local trivialization of the associated bundle $\tilde{\mathfrak{g}}$ to be $X \times \mathfrak{g} \rightarrow X$. The Lagrange-Poincaré operator $\mathcal{L P}$ for a reduced Lagrangian $l$ on $T Q / G=T(Q / G) \oplus \tilde{\mathfrak{g}}$ gives

$$
\mathcal{L P}(l)=\left(\begin{array}{c}
\frac{d}{d t} \frac{\partial l}{\partial \dot{x}^{\alpha}}-\frac{\partial l}{\partial x^{\alpha}}+\frac{\partial l}{\partial \Omega^{a}}\left(B_{\beta \alpha}^{a} \dot{x}^{\beta}+C_{d b}^{a} \Omega^{d} A_{\alpha}^{b}\right) \\
\frac{d}{d t} \frac{\partial l}{\partial \Omega^{b}}-\frac{\partial l}{\partial \Omega^{a}}\left(C_{d b}^{a} \Omega^{d}-C_{d b}^{a} A_{\alpha}^{d} \dot{x}^{\alpha}\right)
\end{array}\right),
$$

where $B_{\beta \alpha}^{a}$ is the curvature of the connection $A_{e}=\left(A_{\alpha}^{a}\right), C_{b d}^{a}$ are the structure constants of the Lie algebra $\mathfrak{g}$, and $\Omega=\left(\Omega^{a}\right)$ and $x=\left(x^{\alpha}\right)$ with $a=1, \ldots, \operatorname{dim} \mathfrak{g}$ and $\alpha=1, \ldots, \operatorname{dim}(Q / G)$. More details of the derivation of (2.9) may be found in sections 3.3 and 4.2 of [17]. In particular, if one chooses a local trivial connection, i.e., $A_{e}=0$, then the Lagrange-Poincaré equation in (2.9) is given by

$$
\mathcal{L P}(l)=\left(\begin{array}{c}
\frac{d}{d t} \frac{\partial l}{\partial \dot{x}^{\alpha}}-\frac{\partial l}{\partial x^{\alpha}} \\
\frac{d}{d t} \frac{\partial l}{\partial \xi^{b}}-C_{d b}^{a} \xi^{d} \frac{\partial l}{\partial \xi^{a}}
\end{array}\right) .
$$

We briefly mention the relation between trajectories of $G$-CL systems and trajectories of RCL systems. Let $(L, F, W)$ be a $G$-CL system and $\left(l,[F]_{G}, W / G\right)$ its RCL system. Choose an arbitrary $G$-equivariant feedback control law $u: T Q \rightarrow$ $W$ for $(L, F, W)$. The control $u$ induces a reduced map $[u]_{G}: T Q / G \rightarrow W / G$. Then, if $(q(t), \dot{q}(t)) \in T Q$ is a trajectory of the closed-loop system $(L, F, u)$, then $\tau_{/ G}(q(t), \dot{q}(t)) \in T Q / G$ is the trajectory of the closed-loop system $\left(l,[F]_{G},[u]_{G}\right)$.

Simple CL systems. We define simple CL systems, which include most mechanical systems in engineering applications.

Definition 2.6. A CL system $(L, F, W)$ on $T Q$ is called simple if its Lagrangian $L: T Q \rightarrow \mathbb{R}$ is of the form kinetic minus potential energy as follows:

$$
L(q, \dot{q})=\frac{1}{2} m_{q}(\dot{q}, \dot{q})-V(q)
$$

where $m$ is a (generalized) mass tensor, i.e., a nondegenerate symmetric (0,2)-tensor. $A$ reduced $C L$ system $\left(l,[F]_{G}, W / G\right)$ is called simple if the reduced Lagrangian $l$ is induced by a $G$-invariant simple Lagrangian $L$ on $T Q$. The acronym $(R) S C L$ will denote "(reduced) simple controlled Lagrangian." 
When a simple $G$-invariant Lagrangian $L$ is given by (2.11), its RSCL $l: T Q / G \rightarrow$ $\mathbb{R}$ is given by

$$
l\left([q, \dot{q}]_{G}\right)=\frac{1}{2}[m]_{G}\left([q, \dot{q}]_{G},[q, \dot{q}]_{G}\right)-[V]_{G}\left([q]_{G}\right),
$$

where $[m]_{G} \in \Gamma\left(Q / G, T^{*} Q / G \otimes T^{*} Q / G\right)$ is the reduced mass tensor induced from the $G$-invariance of the mass tensor $m \in \Gamma\left(Q, T^{*} Q \otimes T^{*} Q\right)$ and $[V]_{G}: Q / G \rightarrow \mathbb{R}$ is the reduced potential energy.

$S C L$-equivalence. We now recall the fundamental definition of CL-equivalence from $[20]$ as follows.

Definition 2.7. Two SCL systems $\left(L_{1}, F_{1}, W_{1}\right)$ and $\left(L_{2}, F_{2}, W_{2}\right)$ are said to be CL-equivalent, or simply, $\left(L_{1}, F_{1}, W_{1}\right) \stackrel{L}{\sim}\left(L_{2}, F_{2}, W_{2}\right)$, if the following EulerLagrange matching conditions hold:

ELM-1: $W_{1}=m_{1} m_{2}^{-1}\left(W_{2}\right)$,

ELM-2: $\operatorname{Im}\left[\mathcal{E} \mathcal{L}\left(L_{1}\right)-F_{1}-m_{1} m_{2}^{-1}\left(\mathcal{E} \mathcal{L}\left(L_{2}\right)-F_{2}\right)\right] \subset W_{1}$,

where $m_{i}$ is the mass tensor of $L_{i}$ and $\operatorname{Im}$ means the pointwise image of the map in brackets.

The following proposition from [20] explains the significance of the CL-equivalence property. It shows that in a very natural sense the two control systems can be made to correspond by using an appropriate choice of control.

Proposition 2.8. Suppose that two $S C L$ systems $\left(L_{i}, F_{i}, W_{i}\right), i=1,2$, are $C L$ equivalent. Then, for an arbitrary control law for one system, there exists a control law for the other system such that the two closed-loop systems produce the same equations of motion. The explicit relation between the two control laws $u_{i}, i=1,2$, is given by

$$
u_{1}=\mathcal{E} \mathcal{L}\left(L_{1}\right)-F_{1}-m_{1} m_{2}^{-1}\left(\mathcal{E} \mathcal{L}\left(L_{2}\right)-F_{2}\right)+m_{1} m_{2}^{-1} u_{2},
$$

where $m_{i}$ is the mass tensor of $L_{i}, i=1,2$.

Proof. Recall that the Euler-Lagrange operator is given by

$$
\mathcal{E} \mathcal{L}(L)(q, \dot{q}, \ddot{q})_{j}=m_{i j} \ddot{q}^{i}+\frac{\partial m_{i j}}{\partial q^{k}} \dot{q}^{i} \dot{q}^{k}-\frac{1}{2} \frac{\partial m_{i k}}{\partial q^{j}} \dot{q}^{i} \dot{q}^{k}+\frac{\partial V}{\partial q^{j}},
$$

where the Lagrangian $L$ is given by

$$
L=\frac{1}{2} m_{i j} \dot{q}^{i} \dot{q}^{j}-V(q) .
$$

One can solve (2.3) for $\ddot{q}$. Denote by $\ddot{q}_{L_{i}}$ the expression of the acceleration $\ddot{q}$ obtained from the closed-loop SCL system $\left(L_{i}, F_{i}, u_{i}\right), i=1,2$. Then,

$$
m_{1}\left(\ddot{q}_{L_{1}}-\ddot{q}_{L_{2}}\right)=u_{1}-m_{1} m_{2}^{-1} u_{2}-\left[\left(\mathcal{E} \mathcal{L}\left(L_{1}\right)-F_{1}\right)-m_{1} m_{2}^{-1}\left(\mathcal{E} \mathcal{L}\left(L_{2}\right)-F_{2}\right)\right] .
$$

The conditions ELM-1 and ELM-2 imply that (2.12) holds if and only if $\ddot{q}_{L_{1}}=\ddot{q}_{L_{2}}$ if and only if they produce the same equations of motion. Notice that the term

$$
\mathcal{E L}\left(L_{1}\right)-m_{1} m_{2}^{-1} \mathcal{E} \mathcal{L}\left(L_{2}\right)
$$

in (2.12) can be regarded as a map defined on $T Q$ because the acceleration $\ddot{q}$ cancels out. 
$R S C L$-equivalence. We now define an equivalence relation among RSCL systems on $T Q / G$.

DeFINITION 2.9. Two RSCL systems $\left(l_{i},\left[F_{i}\right]_{G}, W_{i} / G\right), i=1,2$, are said to be reduced-CL-equivalent (RCL-equivalent), or simply

$$
\left(l_{1},\left[F_{1}\right]_{G}, W_{1} / G\right) \stackrel{L}{\sim}\left(l_{2},\left[F_{2}\right]_{G}, W_{2} / G\right),
$$

if the following reduced Euler-Lagrange matching conditions hold:

RELM-1: $W_{1} / G=\left[m_{1}\right]_{G}\left[m_{2}\right]_{G}^{-1}\left(W_{2} / G\right)$,

RELM-2: $\operatorname{Im}\left[\mathcal{R E} \mathcal{L}\left(l_{1}\right)-\left[F_{1}\right]_{G}-\left[m_{1}\right]_{G}\left[m_{2}\right]_{G}^{-1}\left(\mathcal{R E} \mathcal{L}\left(l_{2}\right)-\left[F_{2}\right]_{G}\right)\right] \subset W_{1} / G$, where $\left[m_{i}\right]_{G}$ is the reduced mass tensor of $l_{i}, i=1,2$, and $\operatorname{Im}$ means the pointwise image of the map in brackets.

Equivalence commutes with reduction. The following theorem explains the relationship between the CL-equivalence relation among $G$-SCL systems and the RCLequivalence relation among RSCL systems.

TheOrem 2.10. Two G-SCL systems are CL-equivalent if and only if their associated RSCL systems are RCL-equivalent.

Proof. Let $(L, F, W)$ be a $G$-SCL system, and $\left(l,[F]_{G}, W / G\right)$ its associated RSCL system. Then, the theorem follows from the $G$-invariance of $W$ and the following relations:

$$
\mathcal{R E} \mathcal{L}(l) \circ \tau_{/ G}^{(2)}=\pi_{/ G} \circ \mathcal{E} \mathcal{L}(L), \quad[F]_{G} \circ \tau_{/ G}=\pi_{/ G} \circ F
$$

where $\tau_{/ G}^{(2)}: T^{(2)} Q \rightarrow T^{(2)} Q / G$ is the $G$-quotient map.

Hence, one can check the RCL-equivalence of two RSCL systems in two ways; one is to directly check it, and the other is to check the CL-equivalence of their associated unreduced $G$-SCL systems. In practice, it is more convenient to check it directly at the reduced level; we shall see an example of this in section 5 .

The following theorem explains the property of the RCL-equivalence relation.

TheOrem 2.11. Suppose that two RSCL systems $\left(l_{i},\left[F_{i}\right]_{G}, W_{i} / G\right), i=1,2$, are $R C L$-equivalent. Then, for an arbitrary control law for one system, there exists a control law for the other system such that the two closed-loop systems produce the same equations of motion. The explicit relation between the two control laws $\left[u_{i}\right]_{G}$, $i=1,2$, is given by

$$
\begin{gathered}
{\left[u_{1}\right]_{G}=\mathcal{R} \mathcal{E} \mathcal{L}\left(l_{1}\right)-\left[F_{1}\right]_{G}-\left[m_{1}\right]_{G}\left[m_{2}\right]_{G}^{-1}\left(\mathcal{R E} \mathcal{L}\left(l_{2}\right)-\left[F_{2}\right]_{G}\right)} \\
+\left[m_{1}\right]_{G}\left[m_{2}\right]_{G}^{-1}\left[u_{2}\right]_{G}
\end{gathered}
$$

where $\left[m_{i}\right]_{G}$ is the reduced mass tensor of $l_{i}, i=1,2$.

Proof. Let $\left[u_{i}\right]_{G}$ be a control for $\left(l_{i},\left[F_{i}\right]_{G}, W_{i} / G\right), i=1,2$. Let $\left(L_{i}, F_{i}, W_{i}\right)$ be the unreduced $G$-CL system of $\left(l_{i},\left[F_{i}\right]_{G}, W_{i} / G\right), i=1,2$. By Theorem 2.10, the two $G$-SCL systems are CL-equivalent. By Proposition 2.8, the two closed-loop $G$-SCL systems $\left(L_{i}, F_{i}, u_{i}\right), i=1,2$, produce the same equations of motion when $u_{1}$ and $u_{2}$ satisfy (2.12). Hence, the two closed-loop RSCL systems $\left(l_{i},\left[F_{i}\right]_{G},\left[u_{i}\right]_{G}\right), i=1,2$, produce the same equations of motion when $\left[u_{1}\right]_{G}$ and $\left[u_{2}\right]_{G}$ satisfy (2.13), because each term in (2.12) is $G$-equivariant. Also notice that, for any choice of $\left[u_{i}\right]_{G}$, one can choose the other $\left[u_{j}\right]_{G}$ such that (2.13) holds.

One can prove Theorem 2.11 by comparing the expressions of "accelerations" of both equations using (2.9), as in the proof of Proposition 2.8. For this purpose, one needs to choose a connection on $Q \rightarrow Q / G$ because one has to split the variations 
to write down the equations of motion in coordinates, because the Euler-Lagrange equations come from the variational principles (see (2.9) of this paper and Chapter 3 of [17] for more detail). In the current proof of Theorem 2.11, we were able to bypass this route by Theorem 2.10 .

3. Reduction of CH systems with symmetry. There is a Hamiltonian counterpart to CL systems called $\mathrm{CH}$ systems. We study the reduction of $\mathrm{CH}$ systems with symmetry in this section.

CH systems. We first recall the definition of $\mathrm{CH}$ systems from [20].

DeFinition 3.1. A CH system is a quadruple $(H, B, F, W)$, where the function $H: T^{*} Q \rightarrow \mathbb{R}$ is called the Hamiltonian, $B \in \Gamma\left(\wedge^{2} T T^{*} Q\right)$ is called an almost Poisson tensor (the main point being that it need not satisfy the Jacobi identity) on $T^{*} Q$, the fiber-preserving map $F: T^{*} Q \rightarrow T^{*} Q$ is called the (external) force map, and the subbundle $W$ of $T^{*} Q$ is called the control subbundle. When a feedback control law $u: T^{*} Q \rightarrow W$ is chosen, the quadruple $(H, B, F, u)$ denotes the closed-loop $C H$ system.

We remark that we choose to use almost Poisson tensors rather than almost symplectic forms, as it is more general and, moreover, is convenient in performing Poisson reduction. The vector field $X_{(H, B, F, u)}$ of a CH system $(H, B, F, W)$ with a control law $u$ is given by

$$
X_{(H, B, F, u)}=B^{\sharp} \mathbf{d} H+\operatorname{vlift}(F)+\operatorname{vlift}(u),
$$

where $\operatorname{vlift}(F)$ and $\operatorname{vift}(u)$ are the vertical lifts defined in (2.1).

Reduction of $G$-invariant $C H$ systems. We define $G$-invariant $\mathrm{CH}$ systems on $T^{*} Q$ and reduced $\mathrm{CH}$ systems on $T^{*} Q / Q$ as follows.

Definition 3.2. Let $G$ be a Lie group action on $Q$. $A$-invariant $\mathrm{CH}(G-\mathrm{CH})$ system is a $C H$ system $(H, B, F, W)$, where $H, B, F$, and $W$ are all $G$-invariant.

Definition 3.3. A reduced $\mathrm{CH}(\mathrm{RCH})$ system is a quadruple $(h, b, f, U)$, where the function $h: T^{*} Q / G \rightarrow \mathbb{R}$ is called the reduced Hamiltonian, $b \in \Gamma\left(\wedge^{2} T\left(T^{*} Q / G\right)\right)$ is called the reduced almost Poisson tensor, the fiber-preserving map $f: T^{*} Q / G \rightarrow$ $T^{*} Q / G$ is called the reduced force map, and the subbundle $U$ of $T^{*} Q / G$ is called the reduced control subbundle.

Suppose that we are given a $G$-CH system $(H, B, F, W)$ on $T^{*} Q$. The $G$-invariant Hamiltonian $H: T^{*} Q \rightarrow \mathbb{R}$ induces the reduced Hamiltonian $h: T^{*} Q / G \rightarrow \mathbb{R}$ as follows:

$$
H=h \circ \pi_{/ G}
$$

The $G$-invariance of the almost Poisson tensor $B \in \Gamma\left(\wedge^{2} T T^{*} Q\right)$ induces a reduced almost Poisson tensor $[B]_{G} \in \Gamma\left(\wedge^{2} T\left(T^{*} Q / G\right)\right)$ as follows: for $f_{1}, f_{2} \in \mathcal{F}\left(T^{*} Q / G\right)$,

$$
[B]_{G_{[q, p]_{G}}}\left(\mathbf{d} f_{1}, \mathbf{d} f_{2}\right)=B_{(q, p)}\left(\pi_{/ G}^{*} \mathbf{d} f_{1}, \pi_{/ G}^{*} \mathbf{d} f_{2}\right) .
$$

This is well defined since

$$
\begin{aligned}
B_{g(q, p)}\left(\mathbf{d}\left(f_{1} \circ \pi_{/ G}\right), \mathbf{d}\left(f_{2} \circ \pi_{/ G}\right)\right) & =B_{(q, p)}\left(g^{*} \mathbf{d}\left(f_{1} \circ \pi_{/ G}\right), g^{*} \mathbf{d}\left(f_{2} \circ \pi_{/ G}\right)\right) \\
& =B_{(q, p)}\left(\mathbf{d}\left(f_{1} \circ \pi_{/ G} \circ g\right), \mathbf{d}\left(f_{2} \circ \pi_{/ G} \circ g\right)\right) \\
& =B_{(q, p)}\left(\mathbf{d}\left(f_{1} \circ \pi_{/ G}\right), \mathbf{d}\left(f_{2} \circ \pi_{/ G}\right)\right)
\end{aligned}
$$

for any $g \in G$, where we used the $G$-invariance of $B$ in the first equality. One can easily check that $[B]_{G}$ is skew-symmetric. The $G$-invariance of $F$ induces the reduced 
force $[F]_{G}: T^{*} Q / G \rightarrow T^{*} Q / G$ satisfying

$$
[F]_{G} \circ \pi_{/ G}=\pi_{/ G} \circ F .
$$

This discussion motivates the following definition.

Definition 3.4. The RCH system of a $G$-CH system $(H, B, F, W)$ is a quadruple $\left(h,[B]_{G},[F]_{G}, W / G\right)$, where $h$ is the reduced Hamiltonian defined in $(3.1),[B]_{G}$ is the reduced almost Poisson tensor defined in (3.2), and $[F]_{G}$ is the reduced force defined in (3.3).

Similarly to Proposition 2.5, the following proposition explains the relations between $G$-CH systems and $\mathrm{RCH}$ systems.

Proposition 3.5. Given a RCH system $(h, b, f, U)$, there is a (not necessarily unique) $G$-CH system $(H, B, F, W)$ whose $R C H$ system is $(h, b, f, U)$.

Proof. Define $H$ by $H=h \circ \pi_{/ G}$. Define a force map $F$ on $T^{*} Q$ as follows: for $\alpha_{q} \in T_{q}^{*} Q, v_{q} \in T_{q} Q$

$$
\left\langle F\left(\alpha_{q}\right), v_{q}\right\rangle=\left\langle f \circ \pi_{/ G}\left(\alpha_{q}\right), \tau_{/ G}\left(v_{q}\right)\right\rangle .
$$

Choose a connection on the principal bundle $T^{*} Q \rightarrow T^{*} Q / G$. (See Chapter 2, Theorem 2.1 in [25] for the proof of the existence.) Then we can split $T T^{*} Q$ into the vertical space $\mathrm{V}$ and the horizontal space $\mathrm{H}$ as $T T^{*} Q=\mathrm{V} \oplus \mathrm{H}$. This induces the decomposition of $T^{*} T^{*} Q$ as $T^{*} T^{*} Q=\mathrm{H}^{\circ} \oplus \mathrm{V}^{\circ}$, where $\mathrm{H}^{\circ}$ and $\mathrm{V}^{\circ}$ are the annihilators of $\mathrm{H}$ and $\mathrm{V}$, respectively. Let hor : $T\left(T^{*} Q / G\right) \rightarrow \mathrm{H}$ be the horizontal lift. Then its dual map hor* $: \mathrm{V}^{\circ} \rightarrow T^{*}\left(T^{*} Q / G\right)$ is an isomorphism. For simplicity, we use $\mathrm{H}^{\circ}$ (resp., $\mathrm{V}^{\circ}$ ) as the projection of $T^{*} T^{*} Q$ onto $\mathrm{H}^{\circ}$ (resp., $\mathrm{V}^{\circ}$ ). Define an almost Poisson tensor $B$ on $T^{*} Q$ as follows: for $\alpha, \beta \in T_{p}^{*} T^{*} Q$

$$
B(\alpha, \beta):=b\left(\text { hor }^{*} \mathrm{~V}^{\circ} \alpha, \text { hor }^{*} \mathrm{~V}^{\circ} \beta\right) .
$$

One can check that this almost Poisson tensor is $G$-invariant. We now show that $\pi_{/ G}: T^{*} Q \rightarrow T^{*} Q / G$ is the Poisson map; i.e., $b=[B]_{G}$. Let $h_{1}, h_{2}$ be two functions on $T^{*} Q / G$. Then $\mathbf{d}\left(h_{i} \circ \pi_{/ G}\right) \in V^{\circ}, i=1,2$. Thus, hor* $\mathbf{d}\left(h_{i} \circ \pi_{/ G}\right)=\mathbf{d} h_{i}, i=1,2$. Hence,

$$
\begin{aligned}
{[B]_{G}\left(\mathbf{d} h_{1}, \mathbf{d} h_{2}\right) } & =B\left(\mathbf{d}\left(h_{1} \circ \pi_{/ G}\right), \mathbf{d}\left(h_{2} \circ \pi_{/ G}\right)\right) \\
& =b\left(\mathbf{d} h_{1}, \mathbf{d} h_{2}\right) .
\end{aligned}
$$

It follows that $[B]_{G}=b$. Let $W=\pi_{/ G}^{-1}(U)$. Then one can see that $(H, B, F, W)$ is a $G$-CH system and that its $\mathrm{RCH}$ system coincides with $(h, b, f, U)$. This completes the proof.

Notice in Proposition 3.5 that there can be more than one $B$ satisfying $[B]_{G}=$ $b$, which is the source of the nonuniqueness of the $G$-CH systems $(H, B, F, W)$ in Proposition 3.5. By Proposition 3.5, we can write an arbitrary RCH system in the form of the $\mathrm{RCH}$ system of a $G$-CH system without loss of generality.

Reduced $C H$ dynamics. Given a $G$-CH system $(H, B, F, W)$, let $\left(h,[B]_{G},[F]_{G}\right.$, $W / G)$ be its RCH system. The (reduced) Hamiltonian vector field $X_{\left(h,[B]_{G},[F]_{G},[u]_{G}\right)}$ of $\left(h,[B]_{G},[F]_{G}, W / G\right)$ with a control $[u]_{G} \in W / G$ is given by

$$
X_{\left(h,[B]_{G},[F]_{G},[u]_{G}\right)}=[B]_{G}^{\sharp} \mathbf{d} h+\operatorname{vlift}\left([F]_{G}\right)+\operatorname{vlift}\left([u]_{G}\right),
$$

where vlift $\left([F]_{G}\right)$ and $\operatorname{vlift}\left([u]_{G}\right)$ are the vertical lifts defined in (2.1). Let $X_{(H, B, F, u)}$ be the vector field of $(H, B, F, W)$ with control $u \in W$. Then we have

$$
X_{\left(h,[B]_{G},[F]_{G},[u]_{G}\right)} \circ \pi_{/ G}=T \pi_{/ G} \cdot X_{(H, B, F, u)} .
$$


The $\mathrm{CH}$-equivalence relation. We shall first recall the $\mathrm{CH}$-equivalence relation among $\mathrm{CH}$ systems on $T^{*} Q$ from [20].

Definition 3.6. Two $C H$ systems $\left(H_{i}, B_{i}, F_{i}, W_{i}\right), i=1,2$, are said to be $\mathrm{CH}-$ equivalent, or simply, $\left(H_{1}, B_{1}, F_{1}, W_{1}\right) \stackrel{H}{\sim}\left(H_{2}, B_{2}, F_{2}, W_{2}\right)$, if the following Hamiltonian matching conditions hold:

HM-1: $W_{1}=W_{2}$,

HM-2: $\operatorname{Im}\left[B_{1}^{\sharp} \mathbf{d} H_{1}+\operatorname{vlift}\left(F_{1}\right)-B_{2}^{\sharp} \mathbf{d} H_{2}-\operatorname{vlift}\left(F_{2}\right)\right] \subset \operatorname{vlift}\left(W_{1}\right)$, where vlift $\left(W_{1}\right)$ is the vertical lift of $W_{1}$ defined in (2.2).

The following proposition explains the significance of the $\mathrm{CH}$-equivalence relation.

Proposition 3.7. Suppose that two CH systems $\left(H_{i}, B_{i}, F_{i}, W_{i}\right), i=1,2$, are $\mathrm{CH}$-equivalent. Then, for an arbitrary control law for one system, there exists a control law for the other system such that the two closed-loop systems produce the same equations of motion. The explicit relation between the two control laws $u_{i}$, $i=1,2$, is given by

$$
\operatorname{vlift}\left(u_{1}\right)=-B_{1}^{\sharp} \mathbf{d} H_{1}-\operatorname{vift}\left(F_{1}\right)+B_{2}^{\sharp} \mathbf{d} H_{2}+\operatorname{vift}\left(F_{2}\right)+\operatorname{vlift}\left(u_{2}\right) .
$$

Proof. Just compare $X_{\left(H_{1}, B_{1}, F_{1}, u_{1}\right)}$ and $X_{\left(H_{2}, B_{2}, F_{2}, u_{2}\right)}$ with controls $u_{1}: T^{*} Q \rightarrow$ $W_{1}$ and $u_{2}: T^{*} Q \rightarrow W_{2}$.

$\mathrm{RCH}$-equivalence. We now introduce an equivalence relation among $\mathrm{RCH}$ systems on $T^{*} Q / G$ as follows.

DeFINITION 3.8. Two RCH systems, $\left(h_{i},\left[B_{i}\right]_{G},\left[F_{i}\right]_{G}, W_{i} / G\right), i=1,2$, are said to be reduced-CH-equivalent (RCH-equivalent), or simply

$$
\left(h_{1},\left[B_{1}\right]_{G},\left[F_{1}\right]_{G}, W_{1} / G\right) \stackrel{H}{\sim}\left(h_{2},\left[B_{2}\right]_{G},\left[F_{2}\right]_{G}, W_{2} / G\right),
$$

if the following reduced Hamiltonian matching conditions hold:

RHM-1: $W_{1} / G=W_{2} / G$,

RHM-2: $\operatorname{Im}\left[\left[B_{1}\right]_{G}^{\sharp} \mathbf{d} h_{1}+\operatorname{vlift}\left(\left[F_{1}\right]_{G}\right)-\left[B_{2}\right]_{G}^{\sharp} \mathbf{d} h_{2}-\operatorname{vlift}\left(\left[F_{2}\right]_{G}\right)\right] \subset \operatorname{vlift}\left(W_{1} / G\right)$, where $\operatorname{vift}\left(W_{1} / G\right)$ is the vertical lift of the subbundle $W_{1} / G$ defined in (2.2).

Reduction commutes with equivalence. The following theorem explains the relation between the RCH-equivalence relation among RCH systems on $T^{*} Q / G$ and the $\mathrm{CH}$-equivalence relation among $G$-CH systems on $T^{*} Q$.

TheOREm 3.9. Two $G-C H$ systems are $C H$-equivalent if and only if their associated $R C H$ systems are $R C H$-equivalent.

Proof. Use Definitions 3.6 and 3.8 as well as the relation (3.5).

TheOREM 3.10. Suppose that two RCH systems $\left(h_{i},\left[B_{i}\right]_{G},\left[F_{i}\right]_{G}, W_{i} / G\right), i=1,2$, are RCH-equivalent. Then, for an arbitrary control law for one system, there exists a control law for the other system such that the two closed-loop RCH systems produce the same equations of motion. The explicit relation between the two control laws $\left[u_{i}\right]_{G}$, $i=1,2$, is given by

$$
\operatorname{vlift}\left(\left[u_{1}\right]_{G}\right)=-\left[B_{1}\right]_{G}^{\sharp} \mathbf{d} h_{1}-\operatorname{vlift}\left(\left[F_{1}\right]_{G}\right)+\left[B_{2}\right]_{G}^{\sharp} \mathbf{d} h_{2}+\operatorname{vlift}\left(\left[F_{2}\right]_{G}\right)+\operatorname{vlift}\left(\left[u_{2}\right]_{G}\right) .
$$

Proof. The proof follows from a straightforward computation using (3.4).

Simple CH systems. Let us review the definition of simple CH systems from [20].

Definition 3.11. A CH system $(H, B, F, W)$ on $T^{*} Q$ is called simple if the Hamiltonian $H$ has the form kinetic plus potential energy,

$$
H(q, p)=\frac{1}{2}\left\langle p, m_{q}^{-1} p\right\rangle+V(q)
$$


and its almost Poisson tensor $B$ is nondegenerate and is of the form

$$
B(q, p)=\left[\begin{array}{cc}
0 & K(q)^{T} \\
-K(q) & J(q, p)
\end{array}\right]
$$

in cotangent coordinates $(q, p)$ on $T^{*} Q$, where $K(q), J(q, p)$ are $n \times n$ matrices with $n=\operatorname{dim} Q$. We call $H$ the simple Hamiltonian and $B$ the simple almost Poisson tensor. The acronym, SCH, denotes "simple controlled Hamiltonian."

One can check that the statement that $B$ has the form (3.6) is independent of the choice of cotangent bundle coordinates on $T^{*} Q$.

Let $B$ be a simple almost Poisson tensor. Then the relation

$$
\operatorname{vlift}\left(\psi_{B}\right)=B \circ \Theta
$$

defines a unique $\psi_{B} \in \Gamma\left(\operatorname{Aut}\left(T^{*} Q\right)\right)$, where $\Theta$ is the canonical 1-form ${ }^{1}$ on $T^{*} Q$ and $B$ is regarded as a bundle map $B: T^{*} T^{*} Q \rightarrow T T^{*} Q$. In other words, there exists a unique $\psi_{B} \in \Gamma\left(\operatorname{Aut}\left(T^{*} Q\right)\right)$ such that the following diagram commutes:

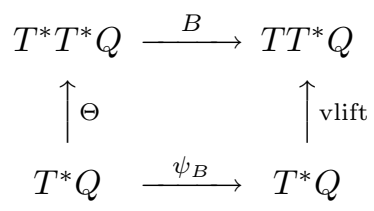

See [20] for the proof of the existence of $\psi_{B}$. When $B$ is given in coordinates as in (3.6), the map $\psi_{B}$ is given in coordinates by

$$
\psi_{B}(q, p)=(q, K(q) p) .
$$

Now, we make the following definition of the reduced simple $\mathrm{CH}$ system.

DeFINITION 3.12. An RCH system $\left(h,[B]_{G},[F]_{G}, W / G\right)$ is called a reduced simple CH system (or RSCH system) if it is the reduced $C H$ system of a $G$-invariant simple CH system.

Simple almost Poisson tensors and their reductions. Recall that in Definition 3.11 we defined simple almost Poisson tensors on $T^{*} Q$ using local coordinates. Here, we characterize the reduced simple almost Poisson tensors using local coordinates, so we may assume that $Q=G \times X$, where $G$ is a Lie group acting on the manifold $X$ trivially. Recall the following identifications by left translation of $G$ :

$$
T^{*} G=G \times \mathfrak{g}^{*}, \quad T T^{*} G=\left(G \times \mathfrak{g}^{*}\right) \times\left(\mathfrak{g} \times \mathfrak{g}^{*}\right), \quad T^{*} T^{*} G=\left(G \times \mathfrak{g}^{*}\right) \times\left(\mathfrak{g}^{*} \times \mathfrak{g}\right) .
$$

We use $\left(g_{a}, \mu_{a}, x_{i}, p_{i}\right)$ as local coordinates for $T^{*} Q=G \times \mathfrak{g}^{*} \times T^{*} X$, and $\left(\mu_{a}, x_{i}, p_{i}\right)$ for $T^{*} Q / G=\mathfrak{g}^{*} \times T^{*} X$, where $a=1, \ldots, \operatorname{dim} G$ and $i=1, \ldots, \operatorname{dim} X$. We will use $\left\{e_{a}\right\}$ as a basis for $\mathfrak{g}$, and $\left\{e_{a}^{*}\right\}$ as its dual basis. Let $B \in \Gamma\left(\wedge^{2} T T^{*} Q\right)$ be a $G$-invariant simple almost Poisson tensor. Then it is of the following form:

$$
\begin{aligned}
B(g, \mu, x, p)= & A_{a b}(x)\left(e_{a} \otimes e_{b}^{*}-e_{b}^{*} \otimes e_{a}\right)+E_{i a}(x)\left(\partial_{x_{i}} \otimes e_{b}^{*}-e_{b}^{*} \otimes \partial_{x_{i}}\right) \\
& +C_{a i}(x)\left(e_{a} \otimes \partial_{p_{i}}-\partial_{p_{i}} \otimes e_{a}\right)+D_{i j}(x)\left(\partial_{x_{i}} \otimes \partial_{p_{j}}-\partial_{p_{j}} \otimes \partial_{x_{i}}\right) \\
& +R_{a b}(\mu, x, p) e_{a}^{*} \otimes e_{b}^{*}+S_{a i}(\mu, x, p)\left(e_{a}^{*} \otimes \partial_{p_{i}}-\partial_{p_{i}} \otimes e_{a}^{*}\right) \\
& +U_{i j}(\mu, x, p) \partial_{p_{i}} \otimes \partial_{p_{j}} .
\end{aligned}
$$

\footnotetext{
$T^{*} Q$.

${ }^{1}$ The canonical 1-form $\Theta$ on $T^{*} Q$ is given by $\Theta=p_{i} \mathbf{d} q^{i}$ in cotangent bundle coordinates $\left(q^{i}, p_{j}\right) \in$
} 
In the matrix form, $B$ is given by

$$
B=\left[\begin{array}{cccc}
O & O & A(x) & C(x) \\
O & O & E(x) & D(x) \\
-A(x)^{T} & -E(x)^{T} & R(\mu, x, p) & S(\mu, x, p) \\
-C(x)^{T} & -D(x)^{T} & -S(\mu, x, p)^{T} & U(\mu, x, p)
\end{array}\right],
$$

where we used the basis for $T_{z} T^{*} Q$ in the following order: $e_{a}, \partial_{x_{i}}, e_{a}^{*}, \partial_{p_{i}}$. The nondegeneracy condition for $B$ is given by

$$
\operatorname{rank}\left[\begin{array}{cc}
A & C \\
E & D
\end{array}\right]=\operatorname{dim} Q
$$

The reduced simple Poisson tensor $[B]_{G}$ is given by

$$
\begin{aligned}
{[B]_{G}(\mu, x, p)=} & E_{i a}(x)\left(\partial_{x_{i}} \otimes e_{b}^{*}-e_{b}^{*} \otimes \partial_{x_{i}}\right)+D_{i j}(x)\left(\partial_{x_{i}} \otimes \partial_{p_{j}}-\partial_{p_{j}} \otimes \partial_{x_{i}}\right) \\
& +R_{a b}(\mu, x, p) e_{a}^{*} \otimes e_{b}^{*}+S_{a i}(\mu, x, p)\left(e_{a}^{*} \otimes \partial_{p_{i}}-\partial_{p_{i}} \otimes e_{a}^{*}\right) \\
& +U_{i j}(\mu, x, p) \partial_{p_{i}} \otimes \partial_{p_{j}} .
\end{aligned}
$$

In a matrix form,

$$
[B]_{G}=\left[\begin{array}{ccc}
R(\mu, x, p) & -E(x)^{T} & S(\mu, x, p) \\
E(x) & O & D(x) \\
-S(\mu, x, p)^{T} & -D(x)^{T} & U(\mu, x, p)
\end{array}\right],
$$

where we used the basis for $T_{[z]}\left(T^{*} Q / G\right)$ in the following order: $e_{a}^{*}, \partial_{x_{i}}, \partial_{p_{i}}$. The nondegeneracy condition for $B$ induces the following rank condition for $[B]_{G}$ :

$$
\operatorname{rank}\left[\begin{array}{ll}
E & D
\end{array}\right]=\operatorname{dim} X .
$$

Remark. One could argue that it might be better if we could characterize all the tensors $b \in \Gamma\left(\wedge^{2} T\left(T^{*} Q / G\right)\right)$ for which there exists a $G$-invariant simple almost Poisson tensor $B$ such that $b=[B]_{G}$. Then we could define RSCH systems without reference to $G$-invariant SCH systems. This point has to be studied more, and we think that the use of connections is crucial; see [31] and [17].

4. Equivalence between reduced simple Lagrangian and Hamiltonian systems. In this section we show the equivalence between the method of RSCL systems on $T Q / G$ and that of RSCH systems on $T^{*} Q / G$.

The $C L-C H$ equivalence theorem. In [19] and [20], the equivalence between the method of SCL systems on $T Q$ and that of SCH systems on $T^{*} Q$ was shown. It is summarized in the following theorem. ${ }^{2}$

THEOREM 4.1. The method of $C L$ systems is equivalent to that of $\mathrm{CH}$ systems for simple mechanical systems in the following sense:

1. For any two simple $C L$ systems $\left(L_{i}, F_{i}^{L}, W_{i}^{L}\right), i=1,2$, there exist two associated simple $C H$ systems ${ }^{3}\left(H_{i}, B_{i}, F_{i}^{H}, W_{i}^{H}\right), i=1,2$, such that

$$
\begin{aligned}
& \left(L_{1}, F_{1}^{L}, W_{1}^{L}\right) \stackrel{L}{\sim}\left(L_{2}, F_{2}^{L}, W_{2}^{L}\right) \\
& \quad \Longleftrightarrow\left(H_{1}, B_{1}, F_{1}^{H}, W_{1}^{H}\right) \stackrel{H}{\sim}\left(H_{2}, B_{2}, F_{2}^{H}, W_{2}^{H}\right) .
\end{aligned}
$$

\footnotetext{
${ }^{2}$ The statement of Theorem 4.1 is slightly different from that of Corollary 4.1 in [20] in that there appears an additional term, $\psi_{B_{2}} \circ \psi_{B_{1}}^{-1}=m_{H_{2}}\left(m_{H_{1}}\right)^{-1}$, in statement 2. However, one can still prove Theorem 4.1 of this paper using section 4 of [20]. For example, see [19] for the proof.

${ }^{3}$ Refer to section 4 of [20] to learn how to find the associated $\mathrm{CH}$ systems.
} 
2. For any two simple $C H$ systems $\left(H_{i}, B_{i}, F_{i}^{H}, W_{i}^{H}\right), i=1,2$, there exist two associated simple $C L$ systems $\left(L_{i}, F_{i}^{L}, W_{i}^{L}\right), i=1,2$, such that

$$
\begin{aligned}
& \left(H_{1}, B_{1}, F_{1}^{H}, W_{1}^{H}\right) \stackrel{H}{\sim}\left(H_{2}, B_{2}, F_{2}^{H}, W_{2}^{H}\right) \\
& \quad \Longleftrightarrow\left(L_{1}, F_{1}^{L}, W_{1}^{L}\right) \stackrel{L}{\sim}\left(L_{2}, F_{2}^{L}, W_{2}^{L}\right) \quad \text { and } \quad \psi_{B_{2}} \circ \psi_{B_{1}}^{-1}=m_{H_{2}}\left(m_{H_{1}}\right)^{-1},
\end{aligned}
$$

with $m_{H_{i}}$ the mass tensor of $H_{i}$ and $\psi_{B_{i}}$ defined in (3.7) for $i=1,2$.

In statement 1 , one does not need the condition $\psi_{B_{2}} \circ \psi_{B_{1}}^{-1}=m_{H_{2}}\left(m_{H_{1}}\right)^{-1}$ along with the $C L$ equivalence condition for the two $C L$ systems, because it automatically holds for the associated $\mathrm{CH}$ systems.

This result implies the following: suppose that we want to find all SCL systems which are equivalent to a given SCL system. One can directly search for them on the Lagrangian side using the CL equivalence relation. Alternatively, one can first find an SCH system which is associated with the given SCL system, secondly search for all $\mathrm{SCH}$ systems which are $\mathrm{CH}$-equivalent to this $\mathrm{SCH}$ system, and finally transform those SCH systems to SCL systems. Those SCL systems are all SCL systems CLequivalent to the original SCL system. In the similar way, one can find all the SCH systems which are CH-equivalent to a given SCH system, directly or with a CLequivalence relation. (Refer to [20] and [19] for more detail.) Hence, one can describe a given simple mechanical system as an SCL system or as an SCH system and then apply the CL method or the $\mathrm{CH}$ method, correspondingly. Both procedures are equivalent.

We now restrict Theorem 4.1 to $G$-invariant systems.

THEOREM 4.2. The method of $G$-invariant $S C L$ systems and that of $G$-invariant $S C H$ systems are equivalent. In other words, Theorem 4.1 restricted to G-invariant simple CL and CH systems holds.

Proof. For the proof, one just needs to keep track of the $G$-invariance in the proof of Theorem 4.1 in [20] and the proof of Theorem 3.2.1 in [19].

The following theorem explains the equivalence between the method of RSCL systems on $T Q / G$ and that of RSCH systems on $T^{*} Q / G$.

THEOREM 4.3. The method of RSCL systems is equivalent to that of RSCH systems in the following sense:

1. For two given $R S C L$ systems $\left(l_{i},\left[F_{i}^{L}\right]_{G}, W_{i}^{L} / G\right), i=1,2$, there exist two associated $R S C H$ systems $\left(h_{i},\left[B_{i}\right]_{G},\left[F_{i}^{H}\right]_{G}, W_{i}^{H} / G\right), i=1,2$, such that

$$
\begin{aligned}
& \left(l_{1},\left[F_{1}^{L}\right]_{G}, W_{1}^{L} / G\right) \stackrel{L}{\sim}\left(l_{2},\left[F_{2}^{L}\right]_{G}, W_{2}^{L} / G\right) \\
& \quad \Longleftrightarrow\left(h_{1},\left[B_{1}\right]_{G},\left[F_{1}^{H}\right]_{G}, W_{1}^{H} / G\right) \stackrel{H}{\sim}\left(h_{2},\left[B_{2}\right]_{G},\left[F_{2}^{H}\right]_{G}, W_{2}^{H} / G\right) .
\end{aligned}
$$

2. For two given RSCH systems $\left(h_{i},\left[B_{i}\right]_{G},\left[F_{i}^{H}\right]_{G}, W_{i}^{H} / G\right), i=1,2$, there exist two associated RSCL systems $\left(l_{i},\left[F_{i}^{L}\right]_{G}, W_{i}^{L} / G\right), i=1,2$, such that

$$
\begin{aligned}
& \left(h_{1},\left[B_{1}\right]_{G},\left[F_{1}^{H}\right]_{G}, W_{1}^{H} / G\right) \stackrel{H}{\sim}\left(h_{2},\left[B_{2}\right]_{G},\left[F_{2}^{H}\right]_{G}, W_{2}^{H} / G\right) \\
& \Longleftrightarrow\left(l_{1},\left[F_{1}^{L}\right]_{G}, W_{1}^{L} / G\right) \stackrel{L}{\sim}\left(l_{2},\left[F_{2}^{L}\right]_{G}, W_{2}^{L} / G\right) \text { and }\left[\psi_{B_{2}} \circ \psi_{B_{1}}^{-1}\right]_{G}=\left[m_{H_{2}}\right]_{G}\left[m_{H_{1}}\right]_{G}^{-1},
\end{aligned}
$$

where $\left[m_{H_{i}}\right]_{G}$ is the reduced mass tensor of $h_{i}$.

Proof. Let us prove statement 1. For given two RSCL systems $\left(l_{i},\left[F_{i}^{L}\right]_{G}, W_{i}^{L} / G\right)$, $i=1,2$, consider their unreduced $G$-SCL systems $\left(L_{i}, F_{i}^{L}, W_{i}^{L}\right), i=1,2$, with $L_{i}=$ $l_{i} \circ \tau_{/ G}$ (see Proposition 2.5). Theorem 4.2 implies that there are two $G$-SCH systems 


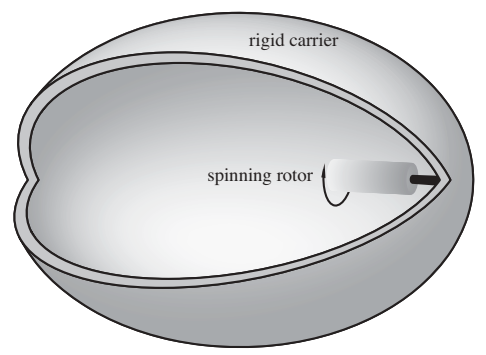

FIG. 5.1. A satellite with a rotor along the third body axis.

$\left(H_{i}, B_{i}, F_{i}^{H}, W_{i}^{H}\right), i=1,2$, such that (4.1) holds. Let $\left(h_{i},\left[B_{i}\right]_{G},\left[F_{i}^{H}\right]_{G}, W_{i}^{H} / G\right)$ be the RSCH system of $\left(H_{i}, B_{i}, F_{i}^{H}, W_{i}^{H}\right)$ for $i=1,2$. Then (4.2) follows from Theorems 2.10 and 3.9 and (4.1). Now we prove statement 2. In this case, use Proposition 3.5 instead of Proposition 2.5, and then proceed in a similar manner.

Hence, one can describe a given simple mechanical system as an SCL/SCH system, apply the CL/CH reduction, and then apply the reduced CL/CH method, correspondingly. Both procedures are equivalent.

Remark. Notice in (4.2) that $\psi_{B_{2}} \circ \psi_{B_{1}}^{-1}$ is $G$-equivariant even though each of $\psi_{B_{i}}$ may not be. This equivariance is a consequence of the following commutative diagram:

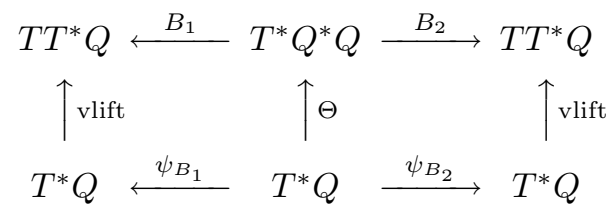

It follows that for $\alpha \in T^{*} Q, B_{2} B_{1}^{-1}(\operatorname{vlift}(\alpha))=\operatorname{vlift}\left(\psi_{B_{2}} \circ \psi_{B_{1}}^{-1}(\alpha)\right)$. One can easily check that vlift is $G$-equivariant, i.e., $\operatorname{vlift}(g \alpha)=g \operatorname{vlift}(\alpha)$ for $g \in G$. Then the $G$ equivariance of $\psi_{B_{2}} \circ \psi_{B_{1}}^{-1}$ follows from the $G$-equivariance of $B_{1}, B_{2}$, and vlift and from the injectivity of vlift.

5. Examples. We review the example of a satellite with a rotor and the EulerPoincaré matching conditions presented in [15] in the framework of the current paper. We then apply the RCL method to the stabilization of the heavy top with rotors. This will show the application of CL systems only. For an excellent application of $\mathrm{CH}$ systems to the problem of underwater vehicle stabilization by internal rotors, refer to [34] and [15].

\subsection{Satellite with a rotor and Euler-Poincaré matching.}

Satellite with a rotor (see [15]). We consider the example of a satellite with a rotor aligned along the third principal axis of the body (see Figure 5.1). The configuration space is $Q=G \times X=\mathrm{SO}(3) \times S^{1}$, with the first factor being the satellite attitude and the second factor being the rotor angle. The Lie group $G=\mathrm{SO}(3)$ acts on the first factor of $Q$ only. We take a trivial (flat) connection on $Q$ such that $T Q / G \simeq \mathfrak{g} \times T X$. Use $\left(\left(\Omega_{1}, \Omega_{2}, \Omega_{3}\right),(\phi, \dot{\phi})\right)$ as coordinates for $\mathfrak{s o}(3) \times T S^{1} \simeq \mathbb{R}^{3} \times T S^{1}$. This system is described by the RSCL system $\left(l_{1},\left[F_{1}\right]_{G}=0, W_{1} / G\right)$ given by

$$
l_{1}(\Omega, \dot{\phi})=\frac{1}{2}\left(\lambda_{1} \Omega_{1}^{2}+\lambda_{2} \Omega_{2}^{2}+\left(I_{3}+J_{3}\right) \Omega_{3}^{3}+2 J_{3} \Omega_{3} \dot{\phi}+J_{3} \dot{\phi}^{2}\right)
$$


and $W_{1} / G=\operatorname{span}\{\mathbf{d} \phi\}$, where $\lambda_{1}>\lambda_{2}>\lambda_{3}:=J_{3}+I_{3}$. Notice that $l_{1}$ does not depend on $\phi$. Recall that the reduced Euler-Lagrange operator $\mathcal{R E} \mathcal{L}$ induces the Lagrange-Poincaré operator $\mathcal{L P}$ in $(2.8)$ with respect to the trivial connection on $Q \rightarrow X$. By (2.10), this Lagrange-Poincaré operator $\mathcal{L} \mathcal{P}\left(l_{1}\right)$ is given by

$$
\mathcal{L P}\left(l_{1}\right)=\left[\begin{array}{c}
\frac{d}{d t} \frac{\partial l_{1}}{\partial \Omega}-\frac{\partial l_{1}}{\partial \Omega} \times \Omega \\
\frac{d}{d t} \frac{\partial l_{1}}{\partial \dot{\phi}}-\frac{\partial l_{1}}{\partial \phi}
\end{array}\right],
$$

where we switched the first and second components of (2.10).

Consider another RSCL system $\left(l_{2}, 0, W_{2} / G\right)$ with $W_{2}=W_{1}$ and

$$
l_{2}(\Omega, \dot{\phi})=\frac{1}{2}\left(\lambda_{1} \Omega_{1}^{2}+\lambda_{2} \Omega_{2}^{2}+\left(I_{3}+J_{3}\right) \Omega_{3}^{3}+2 J_{3} \Omega_{3} \dot{\phi}+\rho J_{3} \dot{\phi}^{2}\right)
$$

with $\rho \in \mathbb{R}$. Here we allow only one free parameter $\rho$ as in [15], but one can consider a more general form of CL system. One can check that RELM-1 and RELM-2 in Definition 2.9 are satisfied. Since these two CL systems are equivalent, one has only to design a controller for the second system, which will give an asymptotically stabilizing controller for the first system by Theorem 2.11. See [8] for the discussion on asymptotic stabilization of the rotation about the middle axis in the body-fixed frame. There, it is shown how to choose $\rho$ and the dissipative input for stability of the equivalent system $\left(l_{2}, 0, W_{2} / G\right)$. This leads to an asymptotically stabilizing controller for the original system $\left(l_{1}, 0, W_{1} / G\right)$.

Euler-Poincaré matching. Here we briefly sketch the proof that the set of EulerPoincaré matching conditions in [12] and [15] is a special case of the reduced EulerLagrange matching conditions in this paper. This set of matching conditions can handle such examples as a satellite with a rotor and underwater vehicles with internal rotors. Let $Q=G \times X$ be the configuration space, where $G$ is a Lie group acting trivially on the manifold $X$. We choose the trivial (flat) connection on $Q \rightarrow X$ to write down the Lagrange-Poincaré equation on $T Q / G \simeq \mathfrak{g} \times T X$ with the Lie algebra $\mathfrak{g}$ of the Lie group $G$. We use $\eta=\left(\eta^{\alpha}\right)$ as coordinates for $\mathfrak{g}$, and $(\theta, \dot{\theta})=\left(\theta^{a}, \dot{\theta}^{a}\right)$ as coordinates for $T X$. By (2.10), the Lagrange-Poincaré operator $\mathcal{L P}$ with respect to the trivial connection is given by

$$
\mathcal{L P}(l)=\left(\begin{array}{c}
\frac{d}{d t} \frac{\partial l}{\partial \eta^{\alpha}}-C_{\gamma \alpha}^{\beta} \eta^{\gamma} \frac{\partial l}{\partial \eta^{\beta}} \\
\frac{d}{d t} \frac{\partial l}{\partial \dot{\theta}^{a}}-\frac{\partial l}{\partial \theta^{a}}
\end{array}\right)
$$

for any reduced Lagrangian $l=l\left(\eta^{\alpha}, \dot{\theta}^{a}, \theta\right)$, where $C_{\gamma \alpha}^{\beta}$ are the structure constants of the Lie algebra $\mathfrak{g}$. In $(5.2)$ we wrote the vertical part of $\mathcal{L P}(l)$ first, while in $(2.10)$ the vertical part was written in the second component.

Let $\left(l, 0, T^{*} X\right)$ be the given RSCL system with the reduced Lagrangian

$$
l\left(\eta^{\alpha}, \dot{\theta}^{a}\right)=\frac{1}{2} g_{\alpha \beta} \eta^{\alpha} \eta^{\beta}+g_{\alpha a} \eta^{\alpha} \dot{\theta}^{a}+\frac{1}{2} g_{a b} \dot{\theta}^{a} \dot{\theta}^{b},
$$

where $g_{\alpha \beta}, g_{\alpha a}, g_{a b}$ are constant functions on $T Q / G$. Notice that this Lagrangian is cyclic in the variables $\theta^{a}$ and that the controls act only on the cyclic variables. Let 


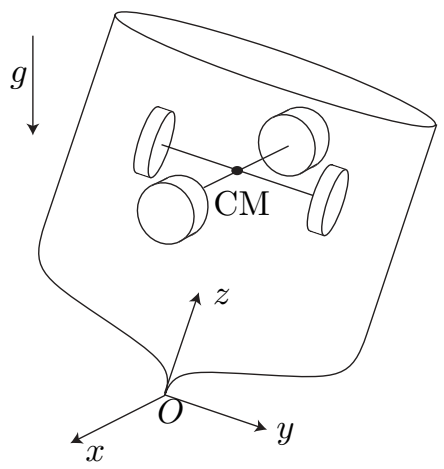

FIG. 5.2. Heavy top with two rotors, each consisting of two rigidly coupled disks. The center of mass is at $C M$.

$\left(l_{\tau, \sigma, \rho}, 0, T^{*} X\right)$ be another RSCL system with the reduced Lagrangian of the following form:

$$
\begin{aligned}
l_{\tau, \sigma, \rho}= & l\left(\eta^{\alpha}, \dot{\theta}^{a}+\tau_{\alpha}^{a} \eta^{\alpha}\right)+\frac{1}{2} \sigma_{a b} \tau_{\alpha}^{a} \tau_{\beta}^{b} \eta^{\alpha} \eta^{\beta} \\
& +\frac{1}{2}\left(\rho_{a b}-g_{a b}\right)\left(\dot{\theta}^{a}+g^{a c} g_{c \alpha} \eta^{\alpha}+\tau_{\alpha}^{a} \eta^{\alpha}\right)\left(\dot{\theta}^{b}+g^{b c} g_{c \beta} \eta^{\beta}+\tau_{\beta}^{b} \eta^{\beta}\right),
\end{aligned}
$$

which is exactly the equation (11) in [15]. See also [14] for the motivation of this choice of the form in (5.3). [15] proposes the following so-called Euler-Poincaré matching conditions:

EP-1: $\tau_{\alpha}^{a}=-\sigma^{a b} g_{b \alpha}$,

EP-2: $\sigma^{a b}+\rho^{a b}=g^{a b}$.

Then one can show that the two assumptions of EP-1 and EP-2 imply the RCLequivalence of the two RSCL systems $\left(l, 0, T^{*} X\right)$ and $\left(l_{\tau, \sigma, \rho}, 0, T^{*} X\right)$. Hence, one can equivalently work with the second system to design controllers. Refer to [15] for the method of constructing a Lyapunov function using the energy and Casimir functions.

5.2. Heavy top with rotors. It is well known that an upright spinning top is unstable if the angular velocity is small. The motion of a heavy top and the stability of the Lagrange top are well studied in [28] and [24]. We use the CL method to asymptotically stabilize the upright spinning motion of a heavy top with small vertical angular velocity, including zero velocity. See Figure 5.2 for the heavy top system. One can notice that the system dynamics are not $\mathrm{SO}(3)$-invariant because the gravitational force breaks the $\mathrm{SO}(3)$ symmetry, and thus we cannot perform the usual reduction of the system by the $\mathrm{SO}(3)$ group. However, there is a way of doing the $\mathrm{SO}(3)$-reduction of this system by considering this system as one depending on a parameter in $\mathbb{R}^{3}$.

In this section, we will first review the general theory of reduction of systems depending on a parameter and then apply this reduction theory to the design of a controller for the heavy top system. We will not develop the whole theory of $C L$ systems depending on a parameter and the reduction theory for those systems with symmetry because it is a straightforward modification of the theory in section 2 of this paper. Moreover, the complete theory of reduction for (uncontrolled) systems depending on a parameter is in [17]. 
Systems depending on a parameter. We here review the reduction theory for systems depending on an advected parameter, as presented in [17]. Consider a Lagrangian

$$
L: T(G \times X) \times V^{*} \rightarrow \mathbb{R},
$$

where $G$ is a Lie group, $X$ is a manifold, and $V^{*}$ is the dual space of the vector space $V$. The value of $L$ at the point $\left(g, x, \dot{g}, \dot{x}, a_{0}\right) \in T(G \times X) \times V^{*}$ will be denoted by $L\left(g, x, \dot{g}, \dot{x}, a_{0}\right)$, as usual, and we will think of $a_{0}$ as a parameter that remains fixed along the evolution of the system. Assume that there is an action of $G$ on $V$, so there is an induced action on $V^{*}$ such that $\left\langle g a_{0}, g b_{0}\right\rangle=\left\langle a_{0}, b_{0}\right\rangle$ for all $a_{0} \in V^{*}$, $b_{0} \in V, g \in G$. Assume that $G$ acts trivially on $X$ and that $L$ is $G$-invariant, i.e.,

$$
L\left(h g, x, h \dot{g}, \dot{x}, h a_{0}\right)=L\left(g, x, \dot{g}, \dot{x}, a_{0}\right)
$$

for $h \in G,\left(g, x, \dot{g}, \dot{x}, a_{0}\right) \in T(G \times X) \times V^{*}$. Define the reduced Lagrangian $l$ : $\mathfrak{g} \times T X \times V^{*} \rightarrow \mathbb{R}$ by

$$
l(\xi, x, \dot{x}, a)=L(e, x, \xi, \dot{x}, a)
$$

for $\xi \in \mathfrak{g},(x, \dot{x}) \in T X, a \in V^{*}$, where $\xi=g^{-1} \dot{g}$ and $a=g^{-1} a_{0}$.

Fix $a_{0} \in V^{*}$. Let $L_{a_{0}}: T(G \times X) \rightarrow \mathbb{R}$ be the restriction of $L$ to $T(G \times X) \times\left\{a_{0}\right\}$. Then, the Euler-Lagrange operator $\mathcal{E} \mathcal{L}\left(L_{a_{0}}\right)$ induces the reduced Euler-Lagrange operator

$$
\mathcal{R E} \mathcal{L}(l): 2 \tilde{\mathfrak{g}} \oplus T^{(2)} X \times T V^{*} \rightarrow \mathfrak{g}^{*} \times T^{*} X
$$

and the equation in (5.6) as follows:

$$
\mathcal{R E} \mathcal{L}(l)=\left(\begin{array}{c}
\frac{d}{d t} \frac{\partial l}{\partial \xi}-\operatorname{ad}_{\xi}^{*} \frac{\partial l}{\partial \xi}-\frac{\partial l}{\partial a} \diamond a \\
\frac{d}{d t} \frac{\partial l}{\partial \dot{x}}-\frac{\partial l}{\partial x}
\end{array}\right)
$$

and

$$
\dot{a}=-\xi a,
$$

where the map $\diamond: V \times V^{*} \rightarrow \mathfrak{g}^{*}$ is defined by $\langle b \diamond a, \eta\rangle=-\langle\eta a, b\rangle$ for $\eta \in \mathfrak{g}, a \in V^{*}$, $b \in V$. One may find the derivation of (5.5) and (5.6) in section 7.4 of [17].

The equations of motion of the reduced Lagrangian $l$ with a $\left(\mathfrak{g}^{*} \times T^{*} X\right)$-valued (reduced) force $f$, are given by

$$
\mathcal{R E} \mathcal{L}(l)=f \quad \text { and } \quad \dot{a}=-\xi a,
$$

where $f$ includes external forces and control forces.

Heavy top with two pairs of rotors. We first describe a heavy top with two pairs of rotors. We mount two pairs of rotors within the top so that each pair's rotation axis is parallel to the first and the second principal axes of the top; see Figure 5.2. Let $I_{1}, I_{2}, I_{3}$ be the moments of inertia of the top in the body-fixed frame. Let $J_{1}, J_{2}$ be the moments of inertia of the rotors around their rotation axes. Let $J_{i 1}, J_{i 2}, J_{i 3}$ be the moments of inertia of the $i$ th rotor with $i=1,2$ around the first, the second, and the third principal axis, respectively. Let $\bar{I}_{1}=I_{1}+J_{11}+J_{21}, \bar{I}_{2}=I_{2}+J_{12}+J_{22}$, 
and $\bar{I}_{3}=I_{3}+J_{13}+J_{23}$. Let $\lambda_{1}=\bar{I}_{1}+J_{1}$ and $\lambda_{2}=\bar{I}_{2}+J_{2}$. Let $M$ be the total mass of the system, $g$ the magnitude of the gravitational acceleration, and $h$ the distance from the origin $O$ to the center of mass of the system.

Let $G=\operatorname{SO}(3), X=S^{1} \times S^{1}, V^{*}=\mathbb{R}^{3}$. We use the following notation for coordinates:

$$
\mathbf{R} \in \mathrm{SO}(3), \quad \theta=\left(\theta_{1}, \theta_{2}\right) \in S^{1} \times S^{1}, \quad a \in \mathbb{R}^{3} .
$$

We will use $\Omega=\left(\Omega_{1}, \Omega_{2}, \Omega_{3}\right) \in \mathbb{R}^{3}$ as coordinates for the Lie algebra $\mathfrak{s o}(3)$ under the Lie algebra isomorphism, $\vee:(\mathfrak{s o}(3),[],) \rightarrow\left(\mathbb{R}^{3}, \times\right)$,

$$
\left(\begin{array}{ccc}
0 & -z & y \\
z & 0 & -x \\
-y & x & 0
\end{array}\right)^{\vee}=(x, y, z)
$$

Let $L: T(G \times X) \times V^{*} \rightarrow \mathbb{R}$ be the Lagrangian defined by

$$
L(\mathbf{R}, \theta, \dot{\mathbf{R}}, \dot{\theta}, a)=\frac{1}{2}\left(\begin{array}{c}
\Omega_{1} \\
\Omega_{2} \\
\Omega_{3} \\
\dot{\theta}_{1} \\
\theta_{2}
\end{array}\right)^{T}\left(\begin{array}{ccccc}
\lambda_{1} & 0 & 0 & J_{1} & 0 \\
0 & \lambda_{2} & 0 & 0 & J_{2} \\
0 & 0 & \bar{I}_{3} & 0 & 0 \\
J_{1} & 0 & 0 & J_{1} & 0 \\
0 & J_{2} & 0 & 0 & J_{2}
\end{array}\right)\left(\begin{array}{c}
\Omega_{1} \\
\Omega_{2} \\
\Omega_{3} \\
\dot{\theta}_{1} \\
\theta_{2}
\end{array}\right)-M g h \mathbf{R}^{-1} a \cdot \chi
$$

where $\Omega=\left(\Omega_{1}, \Omega_{2}, \Omega_{3}\right)=\left(\mathbf{R}^{-1} \dot{\mathbf{R}}\right)^{\vee}$ is the body-fixed angular momentum and $\chi$ is the body-fixed unit vector on the line segment connecting the origin $O$ with the body's center of mass, i.e., $\chi=(0,0,1)$ in the body-fixed frame. follows:

Fix $\mathbf{k}:=(0,0,1) \in \mathbb{R}^{3}$. Let $L_{\mathbf{k}}$ be the restriction of $L$ to $T(G \times X) \times\{\mathbf{k}\}$ as

$$
L_{\mathbf{k}}(\mathbf{R}, \theta, \dot{\mathbf{R}}, \dot{\theta})=L(\mathbf{R}, \theta, \dot{\mathbf{R}}, \dot{\theta}, \mathbf{k})
$$

Then one can check that $L_{\mathbf{k}}$ is the Lagrangian of the heavy top system in Figure 5.2. The actuation is exerted on each pair of rotors, so the control bundle $U$ is given by $U=T^{*} X$.

By (5.4), the reduced Lagrangian $l: \mathfrak{s o}(3) \times T X \times \mathbb{R}^{3} \rightarrow \mathbb{R}$ is given by

$$
\begin{aligned}
l(\Omega, \theta, \dot{\theta}, \Gamma)= & \frac{1}{2}\left(\lambda_{1} \Omega_{1}^{2}+\lambda_{2} \Omega_{2}^{2}+\bar{I}_{3} \Omega_{3}^{2}+2 J_{1} \Omega_{1} \dot{\theta}_{1}+2 J_{2} \Omega_{2} \dot{\theta}_{2}\right) \\
& +\frac{1}{2}\left(J_{1} \dot{\theta}_{1}^{2}+J_{2} \dot{\theta}_{2}^{2}\right)-M g h \Gamma \cdot \chi,
\end{aligned}
$$

where $\Gamma=\left(\Gamma_{1}, \Gamma_{2}, \Gamma_{3}\right)=\mathbf{R}^{-1} \mathbf{k}$. Physically, the vector $\Gamma$ represents the motion of the unit vector with the opposite direction of gravity as seen from the body. Recall that the reduced equations of motion are derived from (5.7) and (5.5).

Let us consider a new reduced Lagrangian $\tilde{l}$ defined by

$$
\begin{aligned}
\tilde{l}(\Omega, \theta, \dot{\theta}, \Gamma)= & \frac{1}{2}\left(\lambda_{1} \Omega_{1}^{2}+\lambda_{2} \Omega_{2}^{2}+\bar{I}_{3} \Omega_{3}^{2}+2 J_{1} \Omega_{1} \dot{\theta}_{1}+2 J_{2} \Omega_{2} \dot{\theta}_{2}\right) \\
& +\frac{1}{2}\left(\rho_{1} J_{1} \dot{\theta}_{1}^{2}+\rho_{2} J_{2} \dot{\theta}_{2}^{2}\right)-M g h \Gamma \cdot \chi,
\end{aligned}
$$

where $\rho_{1}, \rho_{2} \in \mathbb{R}$ are free parameters to be chosen later. See [15] and [21] for the motivation of this choice of the form in (5.8). 
Even though we have not developed the general theory of CL systems depending on a parameter and the associated reduction theory, one can check that Definition 2.9 and Theorem 2.11 hold for reduced CL systems depending on a parameter where one should use the reduced Euler-Lagrange operator in (5.5) instead of (2.9) or (2.10). Notice that the equation in (5.6) is common for all reduced CL systems depending on a parameter. With these modifications in mind, one can check that

$$
\left(l, 0, T^{*} X\right) \stackrel{L}{\sim}\left(\tilde{l}, 0, T^{*} X\right)
$$

By Theorem 2.11, we can work with the RCL system $\left(\tilde{l}, 0, T^{*} X\right)$ to design a controller.

Let us define the angular momentum, $\Pi=\left(\Pi_{1}, \Pi_{2}, \Pi_{2}\right)$, and the control momentum, $\tilde{J}=\left(\tilde{J}_{1}, \tilde{J}_{2}\right)$, as follows:

$$
\begin{aligned}
& \Pi=\frac{\partial \tilde{l}}{\partial \Omega}=\left(\lambda_{1} \Omega_{1}+J_{1} \dot{\theta}_{1}, \lambda_{2} \Omega_{2}+J_{2} \dot{\theta}_{2}, \bar{I}_{3} \Omega_{3}\right), \\
& \tilde{J}=\frac{\partial \tilde{l}}{\partial \dot{\theta}}=\left(J_{1} \Omega_{1}+J_{1} \rho_{1} \dot{\theta}_{1}, J_{2} \Omega_{2}+J_{2} \rho_{2} \dot{\theta}_{2}\right) .
\end{aligned}
$$

By (5.7) and (5.5), the equations of motion of the system $\left(\tilde{l}, 0, T^{*} X\right)$ with a choice of control $v=\left(v_{1}, v_{2}\right)$ are given as follows:

$$
\begin{aligned}
\dot{\Pi} & =\Pi \times \Omega+M g h \Gamma \times \chi, \\
\dot{\tilde{J}} & =v, \\
\dot{\Gamma} & =\Gamma \times \Omega .
\end{aligned}
$$

These dynamics have two constants of motion,

$$
\Pi \cdot \Gamma \text { and }\|\Gamma\|=1,
$$

where $\Pi \cdot \Gamma$ is the vertical component of the space-fixed angular momentum, and $\|\Gamma\|=\left\|\mathbf{R}^{-1} \mathbf{k}\right\|=\|\mathbf{k}\|=1$.

Since the reduced Lagrangian $\tilde{l}$ ( or $l$ ) does not depend on the rotor angle $\theta$, and we are not interested in the angle of rotors but in the angular velocity of rotors, we will remove $X$ from the phase space for the sake of simplicity. Hence, we will regard $\mathfrak{s o}(3) \times \mathbb{R}^{2} \times \mathbb{R}^{3}$ as a new phase space, where $\mathbb{R}^{2}$ is the velocity component of $T X=\mathbb{R}^{2} \times \mathbb{R}^{2}$.

Let $\Omega(0), \dot{\theta}(0)$, and $\Gamma(0)$ with $\|\Gamma(0)\|^{2}=1$ be an initial condition with

$$
\Omega_{3}^{\circ}:=\Pi(0) \cdot \frac{\Gamma(0)}{\bar{I}_{3}}<\sqrt{\frac{M g h}{\bar{I}_{3}}} .
$$

We are interested in the equilibrium $e=\left(\Omega_{e}, \dot{\theta}_{e}, \Gamma_{e}\right)$,

$$
\Omega_{e}=\left(0,0, \Omega_{3}^{\circ}\right), \quad \dot{\theta_{e}}=(0,0), \quad \Gamma_{e}=(0,0,1)
$$

or

$$
\Omega_{e}=\left(0,0, \Omega_{3}^{\circ}\right), \quad \tilde{J}_{e}=(0,0), \quad \Gamma_{e}=(0,0,1),
$$

which corresponds to the upright spinning top with the rotors at rest. Notice that this equilibrium lies in the same level set of $\left(\Pi \cdot \Gamma,\|\Gamma\|^{2}\right)$ as the initial condition. 
We construct a Lyapunov function using the energy-Casimir method (see [8] for more detail of this method). Set

$$
E_{\tilde{\Phi}}=K+U+\Phi\left(\Pi \cdot \Gamma,\|\Gamma\|^{2}\right)+\Psi\left(\tilde{J}_{1}, \tilde{J}_{2}\right),
$$

where the potential energy $U$ is given by $U(\Gamma)=M g h \Gamma \cdot \chi=M g h \Gamma_{3}$, and the kinetic energy $K$ is given by

$$
K=\frac{1}{2}\left(\lambda_{1}-\frac{J_{1}}{\rho_{1}}\right) \Omega_{1}^{2}+\frac{1}{2}\left(\lambda_{2}-\frac{J_{2}}{\rho_{2}}\right) \Omega_{2}^{2}+\frac{1}{2} \bar{I}_{3} \Omega_{3}^{2}+\frac{\tilde{J}_{1}^{2}}{2 J_{1} \rho_{1}}+\frac{\tilde{J}_{2}^{2}}{2 J_{2} \rho_{2}},
$$

in the new coordinates $(\Omega, \tilde{J}, \Gamma)$. Choose the function $\Psi$ as follows:

$$
\Psi\left(\tilde{J}_{1}, \tilde{J}_{2}\right)=\frac{\tilde{J}_{1}^{2}}{2 \epsilon_{1} J_{1}}+\frac{\tilde{J}_{2}^{2}}{2 \epsilon_{2} J_{2}},
$$

where coefficients $\epsilon_{i}$ will be determined later. Choose the function $\Phi$ of the form

$$
\begin{aligned}
\Phi(x, y) & =-\Omega_{3}^{\circ}\left(x-\bar{I}_{3} \Omega_{3}^{\circ}\right)+\frac{1}{2}\left(\bar{I}_{3}\left(\Omega_{3}^{\circ}\right)^{2}-M g h\right)(y-1) \\
& +\frac{1}{2} a_{1}\left(x-\bar{I}_{3} \Omega_{3}^{\circ}\right)^{2}+\frac{1}{2} a_{2}(y-1)^{2},
\end{aligned}
$$

where the constants $a_{1}$ and $a_{2}$ are chosen such that

$$
a_{1}<\frac{-1}{\bar{I}_{3}}
$$

and

$$
4 a_{2}+a_{1}\left(\bar{I}_{3} \Omega_{3}^{\circ}\right)^{2}+\bar{I}_{3}\left(\Omega_{3}^{\circ}\right)^{2}-M g h<\frac{\bar{I}_{3}\left(a_{1} \bar{I}_{3} \Omega_{3}^{\circ}-\Omega_{3}^{\circ}\right)^{2}}{1+a_{1} \bar{I}_{3}} .
$$

One can check that the equilibrium $e$ is a critical point of $E_{\tilde{\Phi}}$. We now find conditions under which this critical point is a local maximum. First, choose $\rho_{i}$ satisfying

$$
\frac{\bar{I}_{3}\left(\Omega_{3}^{\circ}\right)^{2}-M g h}{\left(\Omega_{3}^{\circ}\right)^{2}}<\lambda_{i}-\frac{J_{i}}{\rho_{i}}<0
$$

for $i=1,2$, and then we can choose $\epsilon_{1}$ and $\epsilon_{2}$ such that the second derivative of $E_{\tilde{\Phi}}$ becomes negative definite at $e$, which implies that $E_{\tilde{\Phi}}$ has a local maximum at $e$. For later use, we impose an additional condition on $\rho_{i}$ and $\epsilon_{i}$ as follows:

$$
J_{i}\left(\Omega_{3}^{\circ}\right)^{2}+\left(\epsilon_{i}+\rho_{i}\right)\left(\left(\Omega_{3}^{\circ}\right)^{2}\left(\bar{I}_{3}-\lambda_{i}\right)-M g h\right) \neq 0 .
$$

With (5.19), it is still possible to find $\rho_{i}$ and $\epsilon_{i}$ to ensure negative definiteness of the second derivative of $E_{\tilde{\Phi}}$ at $e$.

The following choice of $v=\left(v_{1}, v_{2}\right)$,

$$
v_{i}=c_{i}\left(\dot{\theta}_{i}+\frac{\tilde{J}_{i}}{\epsilon_{i} J_{i}}\right),
$$

with $c_{i}>0$ for $i=1,2$, implies

$$
\frac{d}{d t} E_{\tilde{\Phi}}=\sum_{i=1}^{2} c_{i}\left(\dot{\theta}_{i}+\frac{\tilde{J}_{i}}{\epsilon_{i} J_{i}}\right)^{2} \geq 0
$$


which proves the Lyapunov stability of the equilibrium $e$ in the closed-loop system. The complete control law $u$ for the original system $\left(l, 0, T^{*} X\right)$ can be obtained from Theorem 2.11.

Asymptotic stabilization will now be shown by using LaSalle's theorem. Since $E_{\tilde{\Phi}}$ has a local maximum at $e$, it is nondecreasing in time, and $\Pi \cdot \Gamma$ and $\|\Gamma\|^{2}$ are conserved, there is a number $c$ such that the set

$$
S=\left\{x \in \mathfrak{s o}(3) \times \mathbb{R}^{2} \times \mathbb{R}^{3} \mid E_{\tilde{\Phi}} \geq c, \Pi \cdot \Gamma=\Pi_{e} \cdot \Gamma_{e},\|\Gamma\|^{2}=1\right\}
$$

is nonempty, compact, and positively invariant. Define

$$
\mathcal{E}=\left\{x \in S \mid \dot{E}_{\tilde{\Phi}}=0\right\}=\{x \in S \mid v=0\} .
$$

Let $\mathcal{M}$ be the largest invariant subset of $\mathcal{E}$. One can show $\mathcal{M}=\{e\}$ by (5.19) after shrinking the set $S$ if necessary. Thus, by LaSalle's theorem, $e$ is asymptotically stable.

Here are the main points in the proof that $\mathcal{M}=\{e\}$. Let $(\Omega(t), \dot{\theta}(t), \Gamma(t))$ be a trajectory in $\mathcal{M}$. The condition $v=0$ and (5.12) imply that $\tilde{J}(t)$ is constant. Hence, $\theta_{i}(t)$ and $\Omega_{i}(t)$ are constant for $i=1,2$. By $(5.9), \Pi_{i}(t), i=1,2$ are constant. Then the third component of (5.11) becomes $\lambda_{3} \dot{\Omega}_{3}(t)=$ constant. By the Lyapunov stability of the equilibrium, it follows $\dot{\Omega}_{3}(t) \equiv 0$. Hence, $\Omega_{3}(t)$ is constant. The first and second component of (5.11) imply that $\Gamma_{1}(t)$ and $\Gamma_{2}(t)$ are constant. Then the third component of (5.13) implies that $\Gamma_{3}(t)$ is constant. So far we have shown that the trajectory $(\Omega(t), \dot{\theta}(t), \Gamma(t))$, or $(\Pi(t), \dot{\theta}(t), \Gamma(t))$, is constant for all $t \geq 0$. Consider the map $f: \mathbb{R}^{8} \rightarrow \mathbb{R}^{10}$ defined by

$$
f(\Omega, \dot{\theta}, \Gamma)=\left(\begin{array}{c}
\Pi \times \Omega+M g h \Gamma \times \chi \\
\Gamma \times \Omega \\
J_{1} \Omega_{1}+\left(\epsilon_{1}+J_{1} \rho_{1}\right) \dot{\theta}_{1} \\
J_{2} \Omega_{2}+\left(\epsilon_{2}+J_{2} \rho_{2}\right) \dot{\theta}_{2} \\
\Pi \cdot \Gamma-\Pi_{e} \cdot \Gamma_{e} \\
\|\Gamma\|^{2}-1
\end{array}\right)=\left(\begin{array}{c}
\dot{\Pi} \\
\dot{\Gamma} \\
\epsilon_{1} v_{1} \\
\epsilon_{2} v_{2} \\
\Pi \cdot \Gamma-\Pi_{e} \cdot \Gamma_{e} \\
\|\Gamma\|^{2}-1
\end{array}\right),
$$

where $\Pi$ is expressed in terms of $(\Omega, \dot{\theta})$ as in (5.9). Then one can see that all the trajectories lying in $\mathcal{M}$ are contained in the set $f^{-1}(O)$. In particular, the equilibrium $\left(\Omega_{e}, \dot{\theta}_{e}, \Gamma_{e}\right)$ in $(5.15)$ is also contained in $f^{-1}(O)$. One can check that the rank of the Jacobian matrix $D f$ at the equilibrium is the full rank 8 by (5.19). Thus, $f$ is locally one-to-one around the equilibrium by Theorem 4.12 in [16]. Therefore, the only possible trajectory totally lying in $\mathcal{M}$ is the equilibrium point itself. It follows from LaSalle's theorem that the equilibrium is asymptotically stable.

Remarks. 1. The above procedure shows that the choice of control gains depends on the initial condition. This is unavoidable because we need to know the value of the constant of motion $\Pi \cdot \Gamma$, which the internal actuation cannot change; however, our suggested controller is robust to small errors in the measurement of the initial condition. Let $\tilde{e}$ be the equilibrium of the form (5.15), with $\tilde{\Omega}_{3}^{\circ}$ instead of $\Omega_{3}^{\circ}$. Suppose the $\Omega_{3}^{\circ}$ used in constructing the control law is very close to the value $\tilde{\Omega}_{3}^{\circ}$. Let $\tilde{E}_{\tilde{\Phi}}$ be the function of the form (5.16), with $\Omega_{3}^{\circ}$ replaced by $\tilde{\Omega}_{3}^{\circ}$. Then $\tilde{e}$ is a critical point of $\tilde{E}_{\tilde{\Phi}}$. By continuity, the second derivative of $\tilde{E}_{\tilde{\Phi}}$ at $\tilde{e}$ will remain negative definite, proving Lyapunov stability of $\tilde{e}$.

2. The same form of controller works for the asymptotic stabilization of the upright spinning top with $\Omega_{3}^{\circ}>\sqrt{M g h / \bar{I}_{3}}$, which is the opposite of (5.14). All 
that needs to be done is to choose $\rho_{i}$ and $\epsilon_{i}$ to make $E_{\tilde{\Phi}}$ have a local minimum at the equilibrium and to choose negative $c_{i}$ such that $E_{\tilde{\Phi}}$ decreases in time. LaSalle's theorem argument guarantees asymptotic stability.

6. Conclusions. In this paper we have studied the reduction of controlled Lagrangian $(\mathrm{CL})$ and controlled Hamiltonian $(\mathrm{CH})$ systems with symmetry. We have shown that the notion of equivalence of controlled systems is preserved by the reduction procedure. This leads to a natural derivation of the Bloch-Leonard-Marsden Euler-Poincaré matching conditions and shows in a precise sense how they are related to the unreduced Euler-Lagrange matching conditions. The theory also shows how to do the equivalent matching on the Hamiltonian side. We studied the examples of a rigid body with rotors (a spacecraft) as well as a heavy top with rotors to illustrate the theory.

In the future we will study more examples and also see to what extent this theory applies to controlled nonholonomic systems with or without symmetry, following [18] and [36], and to degenerate and implicit controlled Lagrangian and Hamiltonian systems, following [6] and [35].

Acknowledgments. The authors thank Hernán Cendra for helpful discussions and the anonymous referees for constructive comments.

\section{REFERENCES}

[1] R. Abraham and J. E. Marsden, Foundations of Mechanics, 2nd ed., Addison-Wesley, Reading, MA, 1978.

[2] R. Abraham, J. E. Marsden, and T. S. Ratiu, Manifolds, Tensor Analysis, and Applications, Appl. Math. 75, 2nd ed., Springer-Verlag, New York, 1988.

[3] D. Auckly, L. Kapitanski, and W. White, Control of nonlinear underactuated systems, Comm. Pure Appl. Math., 53 (2000), pp. 354-369.

[4] D. AuCKLy And L. KAPITANSKI, On the $\lambda$-equations for matching control laws, SIAM J. Control Optim., 41 (2002), pp. 1372-1388.

[5] G. Blankenstein, R. Ortega, and A. J. van Der Schaft, The matching conditions of controlled Lagrangians and interconnection and damping assignment passivity based control, Internat. J. Control, 75 (2002), pp. 645-665.

[6] G. Blankenstein And A. J. van Der Schaft, Symmetry and reduction in implicit generalized Hamiltonian systems, Rep. Math. Phys., 47 (2001), pp. 57-100.

[7] A. M. Bloch, D. E. Chang, N. E. Leonard, and J. E. Marsden, Controlled Lagrangians and the stabilization of mechanical systems II: Potential shaping, IEEE Trans. Automat. Control, 46 (2001), pp. 1556-1571.

[8] A. M. Bloch, D. E. Chang, N. E. Leonard, J. E. Marsden, and C. Woolsey, Asymptotic stabilization of Euler-Poincaré mechanical systems, in Lagrangian and Hamiltonian Methods for Nonlinear Control: A Proceedings Volume from the IFAC Workshop, N. E. Leonard and R. Ortega, eds., Pergamon, Elmsford, NY, 2000, pp. 51-56.

[9] A. M. Bloch, P. S. Krishnaprasad, J. E. Marsden, and G. Sánchez De Alvarez, Stabilization of rigid body dynamics by internal and external torques, Automatica, 28 (1992), pp. $745-756$.

[10] A. M. Bloch, P. S. Krishnaprasad, J. E. Marsden, and T. Ratiu, The Euler-Poincaré Equations and double bracket dissipation, Comm. Math. Phys., 175 (1996), pp. 1-42.

[11] A. M. Bloch, N. E. Leonard, And J. E. Marsden, Stabilization of mechanical systems using controlled Lagrangians, in Proceedings of the 36th IEEE Conference on Decision and Control, San Diego, CA, 1997, pp. 2356-2361.

[12] A. M. Bloch, N. E. Leonard, And J. E. Marsden, Matching and stabilization by the method of controlled Lagrangians, in Proceedings of the 37th IEEE Conference on Decision and Control, Tampa, FL, 1998, pp. 1446-1451.

[13] A. M. Bloch, N. E. Leonard, and J. E. Marsden, Stabilization of the pendulum on a rotor arm by the method of controlled Lagrangians, in Proceedings of the International Conference on Robotics and Automation, Detroit, MI, IEEE, Piscataway, NJ, 1999, pp. 500-505. 
[14] A. M. Bloch, N. E. Leonard, and J. E. Marsden, Controlled Lagrangians and the stabilization of mechanical systems I: The first matching theorem, IEEE Trans. Automat. Control, 45 (2000), pp. 2253-2270.

[15] A. M. Bloch, N. E. Leonard, and J. E. Marsden, Controlled Lagrangians and the stabilization of Euler-Poincaré mechanical systems, Internat. J. Robust Nonlinear Control, 11 (2001), pp. 191-214.

[16] W. M. Boothby, An Introduction to Differentiable Manifolds and Riemannian Geometry, Academic Press, New York, 1986.

[17] H. Cendra, J. E. Marsden, and T. S. Ratiu, Lagrangian reduction by stages, Mem. Amer. Math. Soc., 152 (2001), number 722.

[18] H. Cendra, J. E. Marsden, and T. S. Ratiu, Geometric mechanics, Lagrangian reduction and nonholonomic systems, in Mathematics Unlimited-2001 and Beyond, B. Enquist and W. Schmid, eds., Springer-Verlag, New York, 2001, pp. 221-273.

[19] D. E. Chang, Controlled Lagrangian and Hamiltonian Systems, Ph.D. Thesis, Control and Dynamical Systems Department, California Institute of Technology, Pasadena, CA, 2002.

[20] D. E. Chang, A. M. Bloch, N. E. Leonard, J. E. Marsden, and C. Woolsey, Equivalence of controlled Lagrangian and controlled Hamiltonian systems, ESAIM Control Optim. Calc. Var., 8 (2002), pp. 393-422.

[21] D. E. Chang, and J. E. Marsden, Asymptotic stabilization of the heavy top using controlled Lagrangians, in Proceedings of the 39th IEEE Conference on Decision and Control, Sydney, Australia, 2000, pp. 269-273.

[22] J. Hamberg, General matching conditions in the theory of controlled Lagrangians, in Proceedings of the 39th IEEE Conference on Decision and Control, Phoenix, AZ, 1999, pp. 25192523.

[23] J. Hamberg, Controlled Lagrangians, symmetries and conditions for strong matching, in Lagrangian and Hamiltonian Methods for Nonlinear Control: A Proceedings Volume from the IFAC Workshop, N. E. Leonard and R. Ortega, eds., Pergamon, Elmsford, NY, 2000, pp. $62-67$.

[24] D. D. Holm, J. E. Marsden, And T. S. Ratiu, The Euler-Poincaré equations and semidirect products with applications to continuum theories, Adv. Math., 137 (1998), pp. 1-81.

[25] S. Kobayashi and K. Nomizu, Foundations of Differential Geometry, John Wiley \& Sons, New York, 1963.

[26] P. S. Krishnaprasad, Lie-Poisson structures, dual-spin spacecraft and asymptotic stability, Nonlinear Anal., 9 (1985), pp. 1011-1035.

[27] J. E. Marsden, Lectures on Mechanics, London Math. Soc. Lecture Note Ser. 174, Cambridge University Press, Cambridge, UK, 1992.

[28] J. E. Marsden and T. S. Ratiu, Introduction to Mechanics and Symmetry, 2nd ed., Texts in Appl. Math. 17, Springer-Verlag, New York, 1999.

[29] J. E. MARsden AND J. Scheurle, Lagrangian reduction and the double spherical pendulum, Z. Angew. Math. Phys., 44 (1993), pp. 17-43.

[30] J. E. Marsden And J. Scheurle, The reduced Euler-Lagrange equations, Fields Inst. Commun., 1 (1993), pp. 139-164.

[31] R. Montgomery, J. E. Marsden, and T. S. Ratiu, Gauged Lie-Poisson structures, Contemp. Math., 28 (1984), pp. 101-114.

[32] A. J. VAn DeR Schaft, $L_{2}$-Gain and Passivity Techniques in Nonlinear Control, Communication \& Control Engineering Series, Springer-Verlag, New York, 2000.

[33] L. S. WANG AND P. S. Krishnaprasad, Gyroscopic control and stabilization, J. Nonlinear Sci., 2 (1992), pp. 367-415.

[34] C. Woolsey and N. E. Leonard, Underwater vehicle stabilization by internal rotors, in Proceedings of the American Control Conference, San Diego, CA, 1999.

[35] H. Yoshimura and J. E. Marsden, Variational Principles, Dirac Structure and Implicit Lagrangian Systems, preprint.

[36] D. V. Zenkov, A. M. Bloch, and J. E. Marsden, The Lyapunov-Malkin theorem and stabilization of the unicycle with rider, Systems Control Lett., 45 (2002), pp. 293-302. 